Revue des patrimoines

\title{
Le beurre et la couronne
}

La Tour de Beurre et la Tour couronnée, deux chefs-d'œuvre de la fin du Moyen Age à Rouen

\section{François Verdier}

\section{(2) OpenEdition}

1 Journals

Édition électronique

URL : https://journals.openedition.org/insitu/1148

DOI : $10.4000 /$ insitu. 1148

ISSN : 1630-7305

Éditeur

Ministère de la Culture

\section{Référence électronique}

François Verdier, «Le beurre et la couronne », In Situ [En ligne], 1 | 2001, mis en ligne le 24 avril 2012, consulté le 21 septembre 2021. URL : http://journals.openedition.org/insitu/1148 ; DOI : https:// doi.org/10.4000/insitu. 1148

Ce document a été généré automatiquement le 21 septembre 2021.

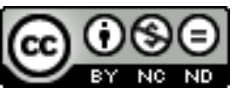

In Situ Revues des patrimoines est mis à disposition selon les termes de la licence Creative Commons Attribution - Pas d'Utilisation Commerciale - Pas de Modification 4.0 International. 


\title{
Le beurre et la couronne
}

\author{
La Tour de Beurre et la Tour couronnée, deux chefs-d'œuvre de la fin du \\ Moyen Age à Rouen
}

François Verdier

\section{NOTE DE L'AUTEUR}

Ce texte préparé à partir de diverses recherches conduites dans le cadre de l'Inventaire général de Haute-Normandie est dédié à Joël Perrin. Celui-ci était souvent venu me rendre visite à Rouen où je lui avais fait découvrir le patrimoine d'une ville du Nord. J'essayais souvent de lui montrer la beauté architecturale des deux grands édifices majeurs de la ville, la cathédrale et Saint-Ouen. Il en était très impressionné mais je sais bien que ce qui l'attirait surtout, c'était la richesse de la peinture religieuse du XVII ${ }^{\mathrm{e}}$ siècle encore très présente dans ces édifices et que nous passions plus de temps devant les retables des chapelles un peu obscures que sur les terrasses et les couronnements des clochers...

1 Cinq ${ }^{1}$ siècles après l'achèvement de la Tour de Beurre de la cathédrale, à peu près contemporaine de la Tour couronnée de l'abbaye de Saint-Ouen, le paysage urbain de Rouen semble définitivement dominé par ces deux chefs-d'œuvre de pierre, seulement surpassés, au-dessus des toits de la ville, par la flèche de bois et de plomb de la Renaissance puis, après sa ruine en 1820 , par celle de fonte de fer du XIX siècle, établies sur la lanterne de la cathédrale ${ }^{2}$. La vue générale de la capitale du duché placée en frontispice du Livre des Fontaines en 1524 nous les présente, toute neuves, au milieu d'une cité au faîte de sa splendeur colorée, au terme d'un demi-siècle d'enrichissement considérable effaçant la période sombre de la guerre de Cent Ans et souriant aux jours heureux de la Renaissance, dans l'euphorie de la découverte des nouveaux mondes et dans la paix d'une nouvelle sociabilité urbaine nourrie de culture humaniste antiquisante. 


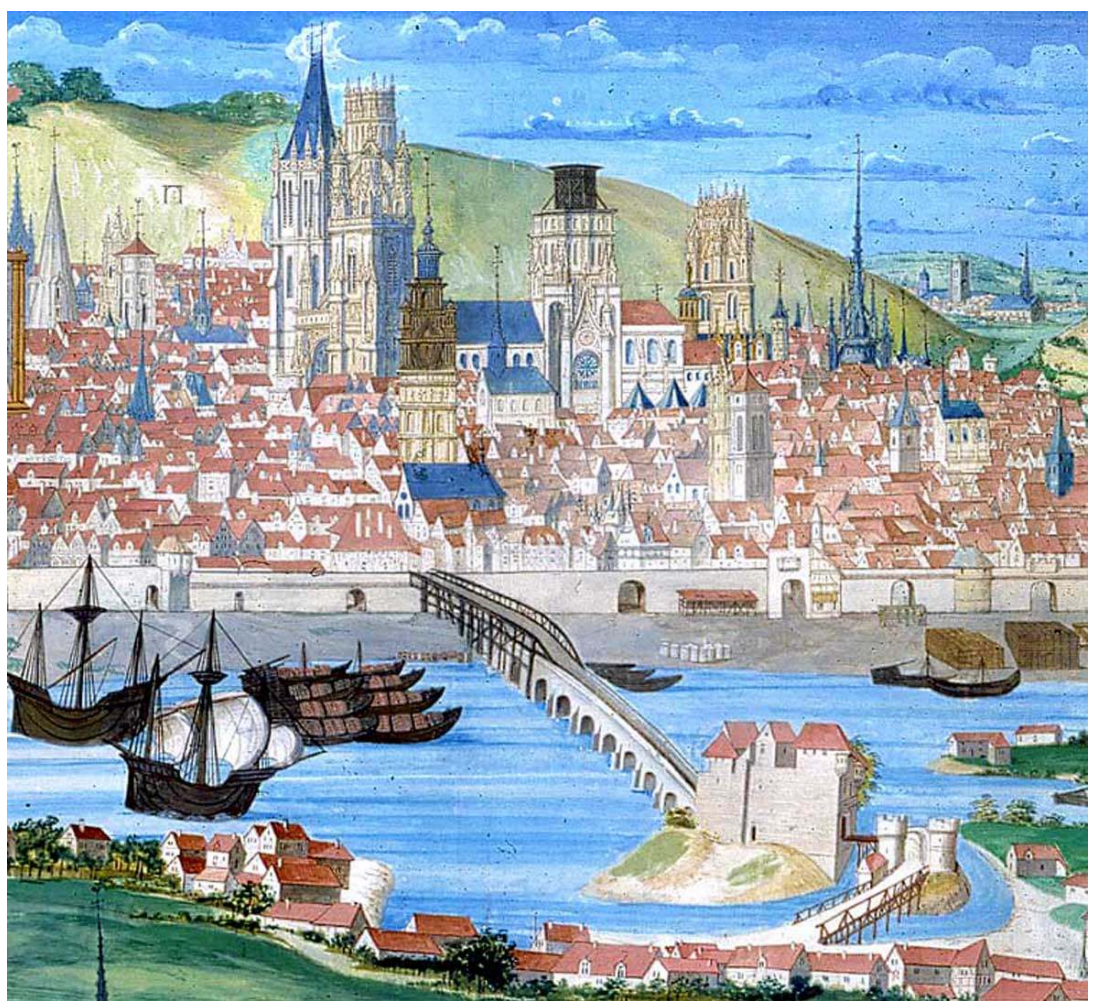

Cathédrale Notre-Dame (Rouen) : la cathédrale Notre-Dame et l'abbaye de Saint Ouen. Extrait du Livre des Fontaines, 1524, Bibl. mun. Rouen

Phot. Inv. D. Trajin, (c) Inventaire général, ADAGP, 1999

2 Deux chantiers majeurs dominent le foisonnement des constructions civiles et religieuses qui, pendant près d'un siècle, témoignent à la fois d'un essor économique et d'une croissance démographique probablement sans autre équivalent dans l'histoire de la ville : tous deux sont des constructions de tours élevées manifestement, nous le verrons ciaprès, dans une concurrence très contrôlée pour parachever l'œuvre des deux principales puissances religieuses de la ville : celle du chapitre et de l'archevêque, avec la Tour de Beurre de la cathédrale, et celle de l'ordre bénédictin et de son abbé avec la Tour couronnée de l'abbaye royale de Saint-Ouen. Ces deux ouvrages sont bien connus comme chefs-d'œuvre d'une époque où les architectes ont élevé nombre de hautes tours et flèches. Mais au moment où la restauration de la Tour de Beurre va s'achever et alors qu'il devient urgent de consolider la tour couronnée de Saint-Ouen, il est intéressant de comparer ce qui rapproche et ce qui distingue ces deux ouvrages rivaux ou complémentaires, que certains visiteurs trop rapides ont peut-être quelquefois confondus et dont chaque habitant de la ville se demande quotidiennement, depuis bientôt cinq cents ans, lequel est le plus fantastique.

3 La renommée de la Tour de Beurre vient probablement en premier lieu de son nom légendaire attaché au monument le plus célèbre de la ville. Depuis le début du XIX siècle, pas un guide n'omet de signaler qu'elle doit son nom aux fortunes qu'il a fallu rassembler pour l'édifier et qui proviennent des aumônes collectées par le chapitre en dispense pour consommer du beurre durant le carême ${ }^{3}$. La légende est sympathique et correspond bien à la fois à une certaine vision de l'Eglise de la fin du Moyen Age comme à la bonne chère 
coutumière depuis longtemps en Normandie. Il n'est pas certain que cette légende résisterait définitivement à la critique des historiens actuels, mais le fait est que la construction de la tour fut particulièrement dispendieuse et l'ouvrage financé par le chapitre sans que l'argent ait semblé manquer (du moins jusque vers 1507 où, comme on le verra, le projet de flèche n'est pas mis en œuvre).

Figure 2

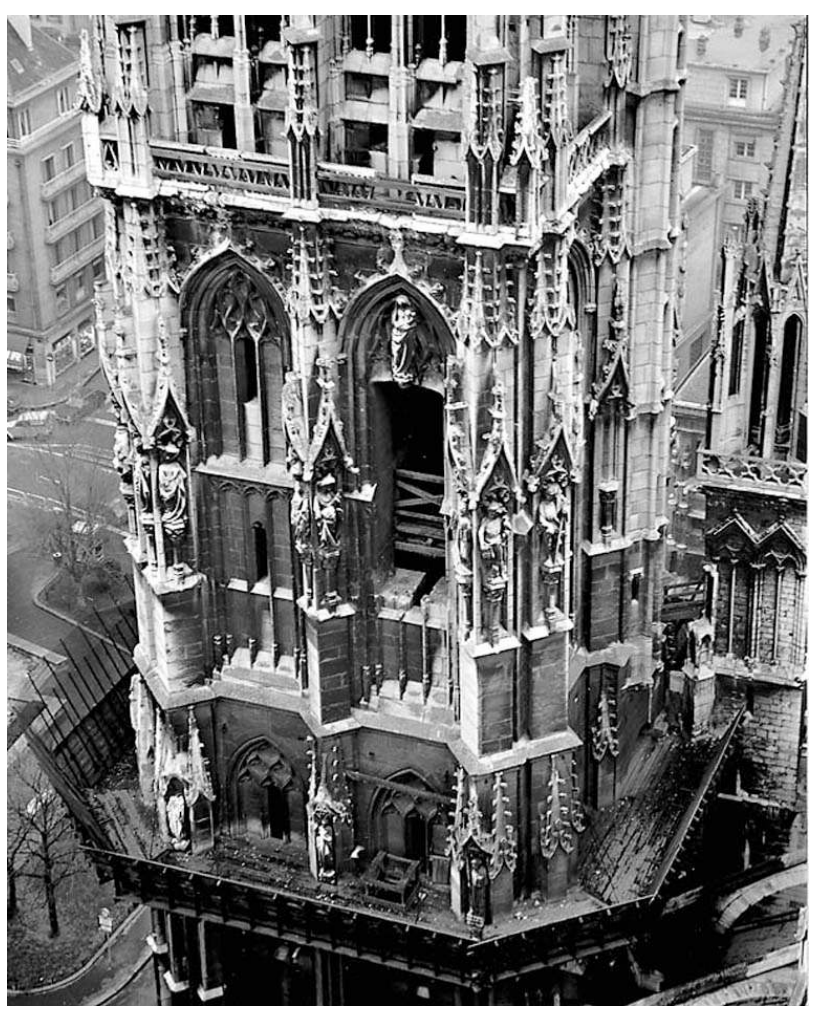

Cathédrale Notre-Dame (Rouen) : Tour de Beurre, élévation extérieure est Phot. Inv. C. Kollmann, (c) Inventaire général, ADAGP, 1981 
Figure 3

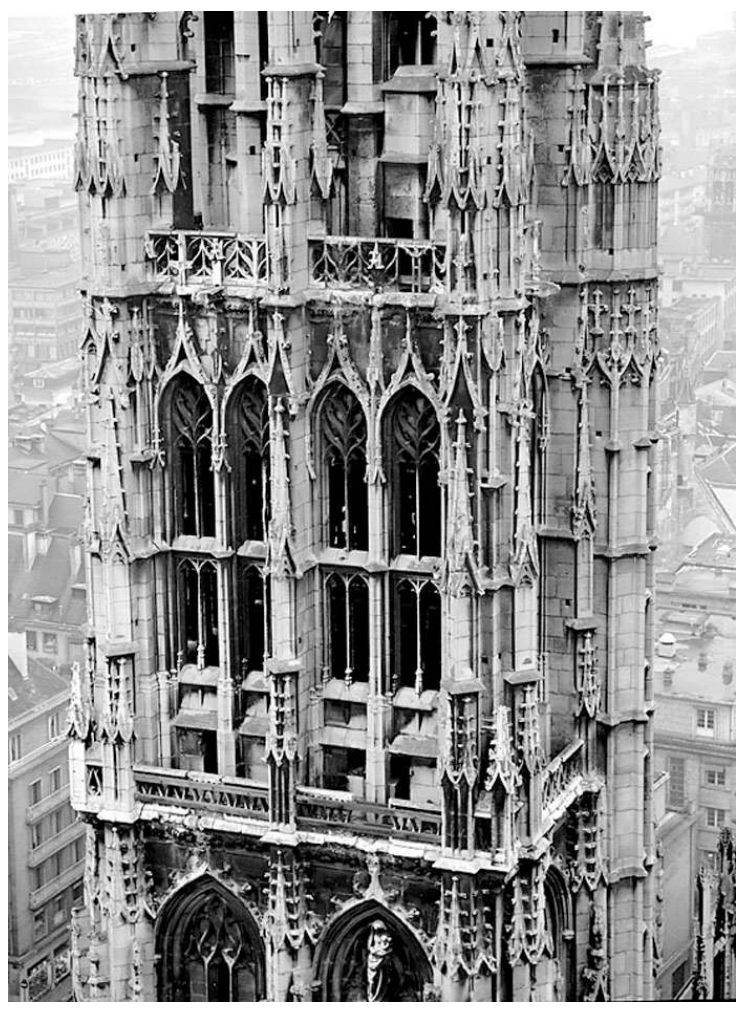

Cathédrale Notre-Dame (Rouen) : Tour de Beurre, élévation extérieure est Phot. Inv. C. Kollmann, (c) Inventaire général, ADAGP, 1981 


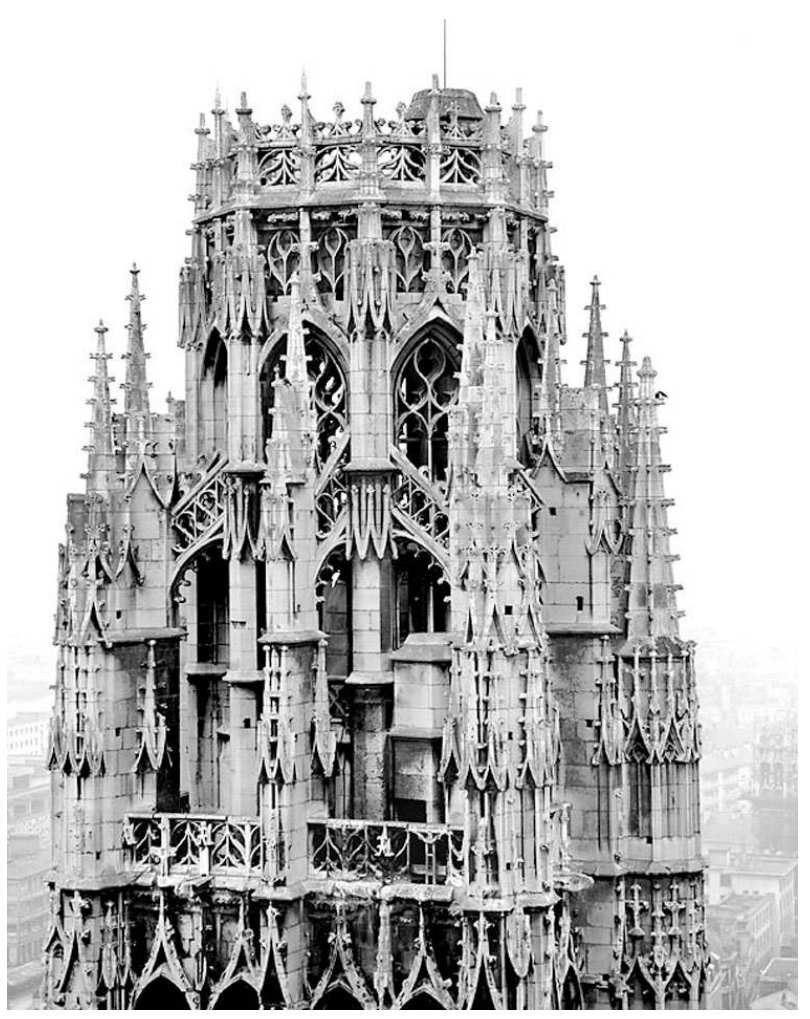

Cathédrale Notre-Dame (Rouen) : Tour de Beurre, élévation extérieure est

Phot. Inv. C. Kollmann, (c) Inventaire général, ADAGP, 1981

Notice de la base Mérimée

L'autre raison majeure de l'admiration portée à cette tour est la célébrité de la cathédrale elle-même et de son étonnante façade en particulier où, dernière pièce rapportée, la tour joue un rôle essentiel et concourt à équilibrer le gigantesque écran de pierre palliant l'absence de façade de l'édifice du XIII ${ }^{\mathrm{e}}$ siècle. Certes, la façade d'une cathédrale idéale ne saurait se concevoir, depuis Viollet-le-Duc, mais surtout depuis les grands modèles carolingiens ou romans, sans " composition harmonique » à deux tours. Mais ce n'était précisément pas le cas à Rouen où la tour nord, la tour Saint-Romain, vestige isolé de la cathédrale antérieure, est mal reliée à l'édifice gothique, et la tour sud, la Tour de Beurre, est une implantation hors œuvre qui complète bien tardivement un édifice certes imposant dans la ville médiévale, mais dont le parti occidental reste bien peu cohérent.

Il faut effectivement, par exemple, essayer de s'imaginer le parvis de Notre-Dame tel que l'avait vu Jeanne d'Arc en 1431. Au nord, fermant le quartier du chapitre, la tour SaintRomain avec son premier niveau aveugle et massif et ses niveaux de salle haute et de beffroi non encore exhaussé et constituant alors la seule chambre des cloches. Au milieu, un ensemble centré de trois portails couverts de cintres assez massifs butant la nef sous des fenêtres hautes et des pignons portant un modeste réseau d'arcatures et de galeries de rois. Plus au sud encore, contre la nef, une église paroissiale, Saint-Etienne, dont on ne connaît ni la disposition ni l'élévation, mais qui ne devait pas magnifier la monumentalité d'une cathédrale dont les splendeurs et la cohérence restent plus évidentes au transept, à la lanterne ou surtout autour du sanctuaire. 
6 L'édification de la tour, confiée par le chapitre, en 1487, à l'architecte rouennais Guillaume Pontifs dure une trentaine d'années, poursuivie à partir de 1501 par Jacques Le Roux, autre célèbre architecte de la ville. Considéré globalement, l'ensemble est à la fois autonome et très cohérent, s'élevant d'un jet à $77 \mathrm{~m}$ sur une base carrée de $12 \mathrm{~m}$ environ, soit un rapport de 1 à 6 , ce qui indique son caractère exceptionnellement élancé et son remarquable équilibre. Sa position est privilégiée, en avant-poste au sud-ouest, elle domine toute la cathédrale (si l'on ne tient pas compte des croix de fer de la tour SaintRomain, ni du fait qu'au moment de sa construction, la lanterne n'était pas amortie par la flèche dressée en 1545 par Robert Becquet). Elle marque également un nouveau rapport à la ville et à son port sur le fleuve. L'ancien axe majeur de la cathédrale, celui du XI ${ }^{\mathrm{e}}$ ou du $\mathrm{XII}^{\mathrm{e}}$ siècle, reliait par la rue de l'Epicerie le portail de la Calende à la Fierte, pas encore l'édifice actuel qui sera bâti en 1542 mais l'ancienne chapelle du palais ducal dans laquelle l'archevêque libérait chaque année un condamné à mort en mémoire de saint Romain et en signe de privilège particulier sur l'aristocratie de la ville. La nouvelle façade et sa tour dominent le port et les quartiers commerçants de la bourgeoisie pour faire sonner plus haut et plus loin le carillon de l'Ave Maris Stella appelant à la prière et à l'intercession divine.

7 L'histoire détaillée de la construction de cette tour est bien établie depuis les travaux publiés en 1905 par Charles de Robillard de Beaurepaire, archiviste départemental, à partir du dépouillement de la série G. En 1485, l'archevêque Robert de Croixmare et le chapitre, particulièrement bien disposés vis-à-vis de l'architecte Guillaume Pontifs qui vient d'achever pour eux des travaux considérables et magnifiques à la tour SaintRomain, à l'escalier de la librairie, au pourtour du choeur et aux fenêtres hautes, décident de lui confier l'édification d'une tour remarquable qui fasse la fierté de la ville entière. Architecte consacré, il mène le chantier avec plus ou moins de régularité presque jusqu'à sa mort en 1497. On ne sait pas exactement à quel stade se trouvait alors son travail mais Saint-Etienne-la-Grande-Eglise était probablement achevée dès 1489. Jacques Le Roux, autre architecte fameux dans la ville et alors déjà âgé, se voit confier en 1496 l'achèvement de la tour. Il fait vérifier l'état des fondations et reprendre, avec un luxe de raffinement que lui reproche le chapitre, l'élévation et la décoration des parties hautes. En 1499, parvenu au faîte du beffroi, le chantier s'interrompt à nouveau et le chapitre délibère sur le couronnement à choisir : terrasse ou aiguille ? L'état dégradé des finances, la discorde avec l'architecte, l'implication du cardinal Georges d'Amboise, l'avis demandé aux experts, tout empêche la reprise du chantier. La consultation s'éternise, les projets contradictoires sont écartés par la ville, par les experts, par le cardinal, jusqu'à ce qu'en 1505 tout le monde s'accorde sur un énième projet de flèche. Pourtant, celle-ci ne fut pas construite et Beaurepaire en voit la cause dans les importants désordres apparus aux voûtes de la nef qui mobilisent dans l'urgence les ressources du chapitre. On s'en tiendra à une "couronne ", formule sans précédent, peut-être dérivée du projet de flèche, probablement liée à la nécessité de limiter la dépense et réalisée par un architecte dans les dernières années d'une très brillante carrière (il sera inhumé, privilège rare, dans le transept de la cathédrale en 1510). On verra cependant plus loin que la couronne, loin de nous paraître aujourd'hui la marque d'une flèche avortée, constitue la partie la plus géniale de cette tour.

8 Le programme architectural de la tour est triple, du moins si l'on s'en tient au parties réalisées : au rez-de-chaussée, une église paroissiale, au premier étage une vaste salle, au second, particulièrement développé, une chambre à cloches. L'ouvrage de couronnement 
n'a pas de fonction particulière si ce n'est que, parfaitement accessible, il donne à contempler depuis une coursière sommitale le spectacle de la ville au milieu d'une forêt de clochetons et de pinacles...

9 Tout en bas, l'église Saint-Etienne paraît assez mal installée. Eglise paroissiale incluse dans la cathédrale, son plan massé, l'encombrement des piles massives de la tour, les hauts murs empâtés en glacis en font un espace sombre et pesant dans lequel le mobilier liturgique trouve mal sa place, un volume sans circulation, sans accès monumental. Ce parent pauvre du chapitre a néanmoins reçu un bel ensemble de verrières. Peut-être l'abandon de la fonction paroissiale concourt-il à l'impression d'espace annexe obscur et inutile, seulement habité aujourd'hui par un ensemble de pierres tombales et de monuments votifs oubliés derrière les grilles fermées de la clôture de nef.

10 La salle de l'étage carré est fort belle avec sa voûte d'ogives à huit branches et clef annulaire. Les parements, la sculpture des culots, les fenêtres petites mais nombreuses témoignent du raffinement de la mise en œuvre de ces salles hautes dont la fonction reste énigmatique. Il n'y a pratiquement pas d'accès à cet étage greffé sur le pourtour de la nef, et le seul usage connu est d'y avoir remisé les réserves de plomb de couverture, sorte de trésor architectural stocké de façon certes rationnelle au niveau des toitures de la nef, et ainsi protégé du vol, mais sans rapport avec la qualité de mise en œuvre de ce lieu élevé.

Figure 5

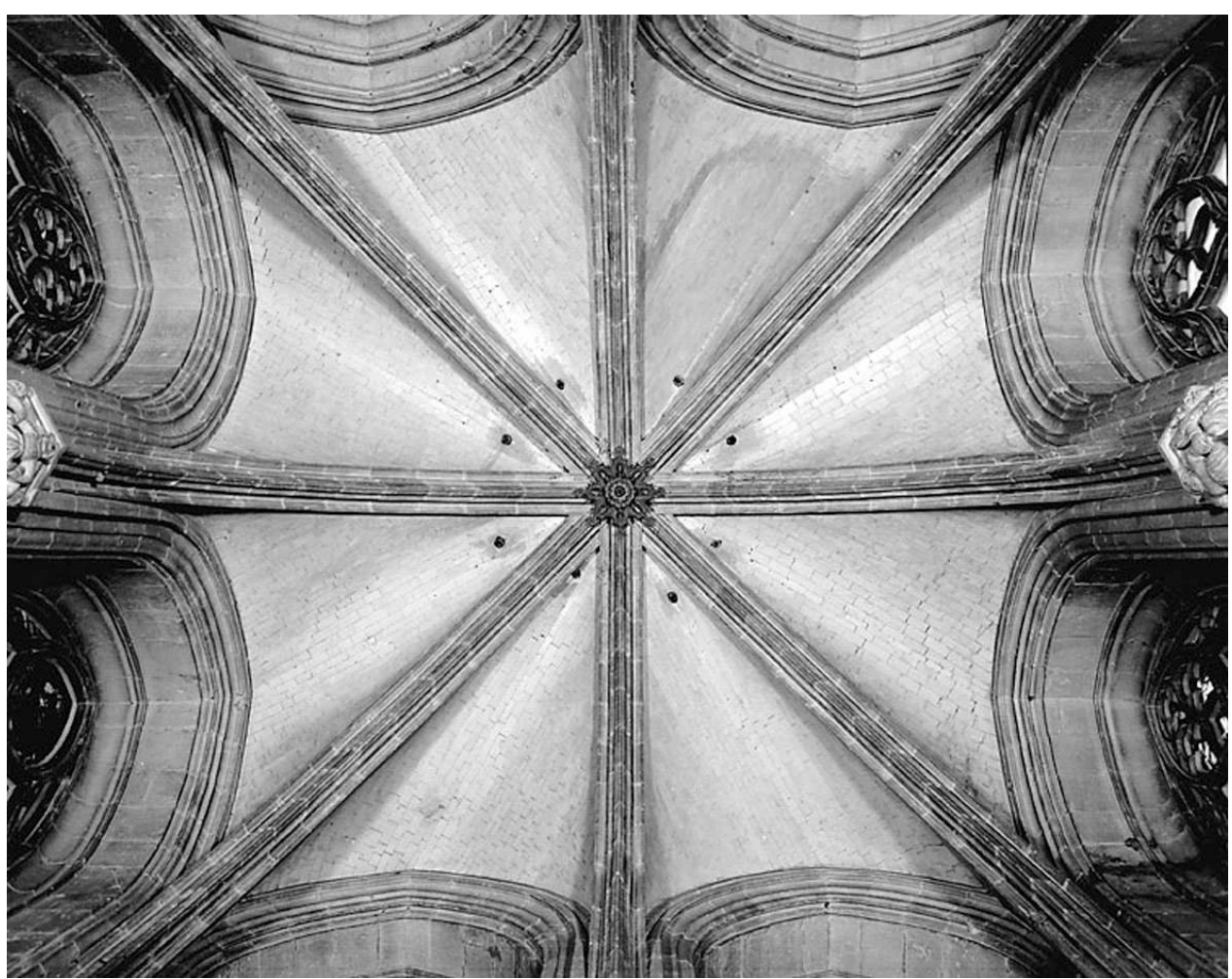

Cathédrale Notre-Dame (Rouen) : Tour de Beurre, élévation intérieure, voûtes du rez-de-chaussée Phot. Inv. C. Kollmann, (c) Inventaire général, ADAGP, 1986

11 La chambre des cloches forme un volume impressionnant, surtout telle qu'elle apparait aujourd'hui, vidée de son beffroi. Elle monte sur cinq niveaux et superposait en fait trois beffrois comprenant des cloches de toutes tailles depuis le fameux bourdon de Georges d'Amboise, de 36000 livres, installé en 1501 et détruit en 1786, jusqu'à nombre de cloches 
plus petites et surtout un carillon dont l'importance a varié suivant les époques, mais qui comprenait encore récemment une soixantaine de cloches.

La présentation de la structure de cette tour est particulièrement intéressante. On a discuté de la qualité de ses fondations, très souvent le point faible des tours. Le premier architecte n'avait peut-être pas imaginé la hauteur ni la charge exacte de l'ouvrage. Il lui était difficile, également, de fouiller trop largement au pied même de la nef de la cathédrale. Toujours est-il que la tour penche un peu vers le sud, du moins dans ses premiers niveaux. Rien d'assez sensible pour lui apporter la célébrité de sa consoeur de Pise, mais suffisamment pour inquiéter le chapitre, faire expertiser l'ouvrage, arrêter le chantier en 1489 et prendre des mesures pour rééquilibrer et redresser les niveaux supérieurs.

En réalité, la tour monte (fig. $\mathbf{n}^{\circ} \mathbf{6}$ ) de fond comme un tube carré de pierre parfaitement appareillé, raidi par un ensemble continu de contreforts, jumelés sur les angles et simples au milieu de chaque face, allégé dans sa partie supérieure par des percements, généreux et également répartis, et divisé horizontalement par trois niveaux de voûtes agissant comme voiles pour répartir les charges et stabiliser les poussées. De fait, une telle structure a parfaitement résisté à cinq siècles d'exposition à toutes sortes de contraintes (jusqu'aux bombardements de la Seconde Guerre mondiale qui ont détruit à ses pieds le collatéral sud de la nef), sans nécessiter de reprise de gros œuvre, sans porter de marque de rupture, la seule fissure décelable correspondant à une maladroite trouée ouverte vers 1501 pour le passage du bourdon de Georges d'Amboise.

Figure 6

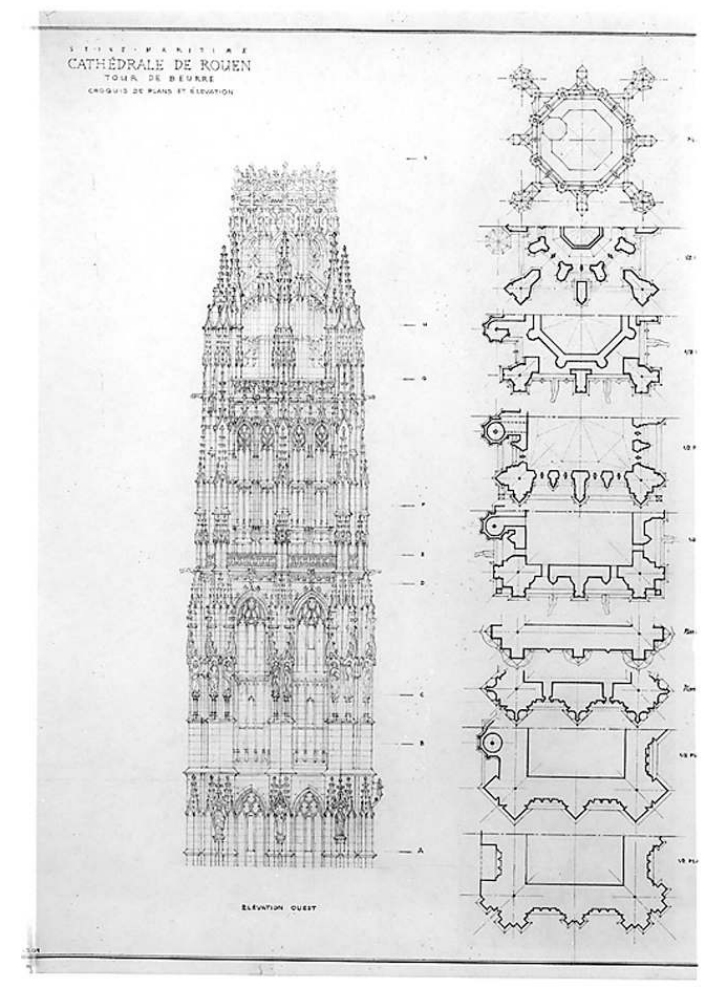

Cathédrale Notre-Dame (Rouen) : Tour de Beurre, croquis de plan et d'élévation ouest. Relevé par Chauvel, 1954. Médiathèque du Patrimoine, Paris

Phot. E. Badie Modini (c) Médiathèque du patrimoine 
Figure 7

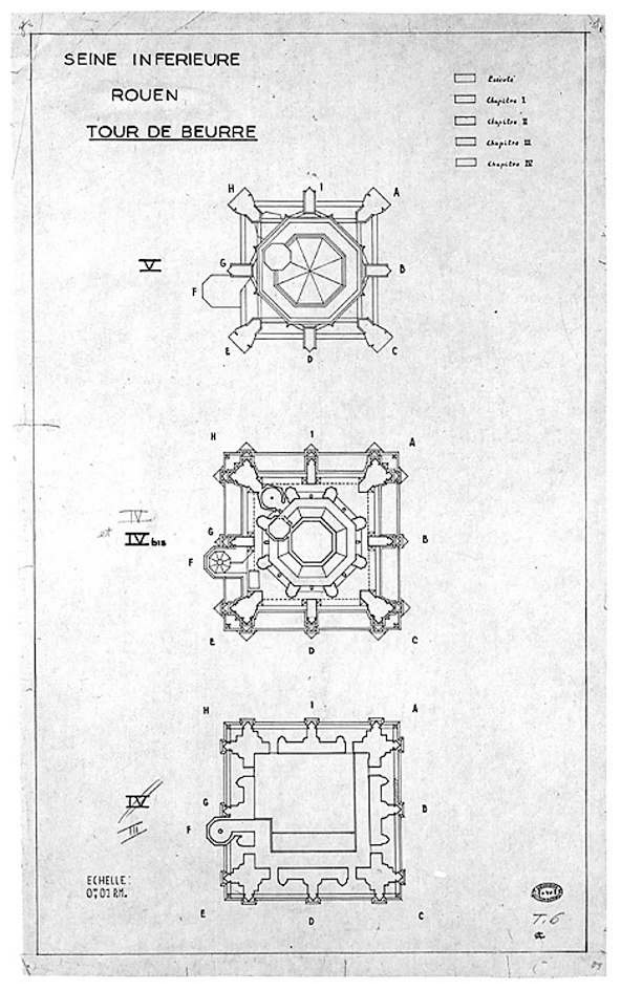

Cathédrale Notre-Dame (Rouen) : Tour de Beurre, plan au niveau du couronnement. Relevé par Chauvel, 1950. Médiathèque du Patrimoine, Paris

Phot. E. Badie Modini (c) Médiathèque du patrimoine

Le plan carré (fig. $\mathbf{n}^{\circ}$ 7), d'une parfaite géométrie, se superpose à chaque étage avec ses doubles contreforts d'angle et ses contreforts médians jusqu'au sommet du beffroi couvert d'une coupole octogonale sur trompes. A ce niveau, la structure se dédouble. Une coupole intérieure (fig. $\mathbf{n}^{\circ} \mathbf{8}$ ), sorte de voûte de cloître de plan octogonal, couvre le volume, charge fortement les murs d'enveloppe et porte sur ses reins les huit supports du couronnement octogonal. La charge importante de ce couronnement est reprise et épaulée par un ensemble de seize arcs boutants jumelés. Là s'affiche le véritable talent de l'architecte, Jacques Le Roux, capable à la fois de concevoir les formes, les tracés et les équilibres d'un ensemble parfaitement harmonieux et logique, mais aussi de conduire le travail des tailleurs de pierre (fig. $\mathbf{n}^{\circ}$ 9) : la stéréotomie de l'ensemble est prodigieuse, virtuose même lorsqu'il s'agit de lier par des arcs biais le plan carré de l'enveloppe et l'octogone de la couronne centrale. Si celui-ci a dû renoncer à l'édification d'une flèche, nous pouvons aujourd'hui ne pas trop le regretter car celle-ci n'aurait probablement pas résisté jusqu'à notre époque $e^{4}$. La structure complètement évidée se réduit à un jeu d'organes de supports, d'arcs-boutants et de pinacles de charge disposés sur les pans droits et biais pour former, selon des angles et des pentes aigus, un ensemble centré et triangulé, parfaitement raidi, mais dont les lignes sont étonnamment souples, formées de courbes accentuées par des évidements moulurés en soufflets, particulièrement au niveau des balustrades ou dans les innombrables pinacles qui ponctuent les assises supérieures. On a surtout retenu, le chapitre le premier qui en contestait la dépense, la richesse de l'ornementation sculptée qui fait vibrer la pierre et donne toute sa transparence à cette structure déjà aérienne. On peut être tout autant séduit, aujourd'hui, par le graphisme et 
l'harmonie des lignes qui s'élèvent comme un contrepoint et, dans un travail qui évoque l'orfèvrerie ou la dentelle, fuient dans un enchevêtrement magique.

Figure 8

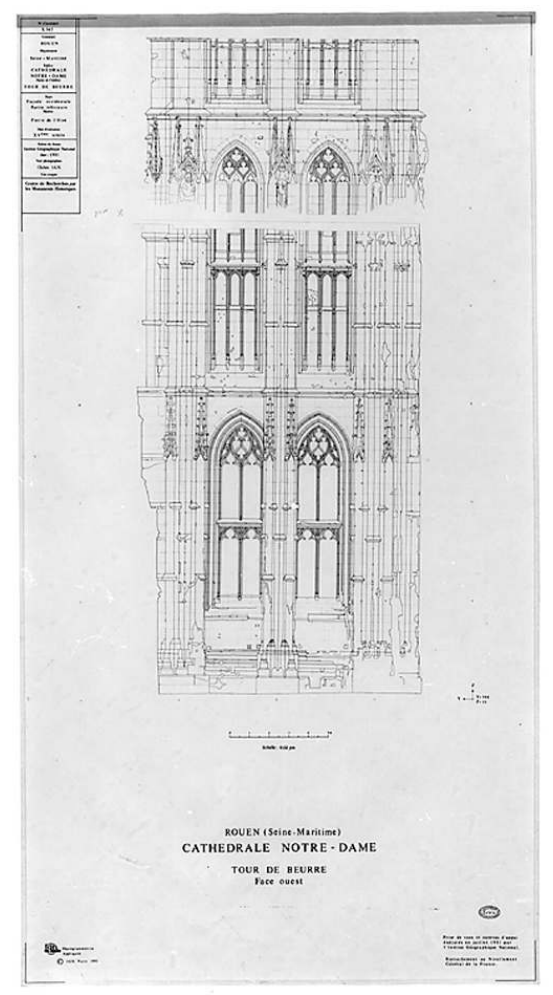

Tour de Beurre, élévation extérieure Sud, niveaux inférieurs. Relevé photogrammétrique I.G.N., 1981. Médiathèque du Patrimoine, Paris

Phot. E. Badie Modini (c) Médiathèque du patrimoine 
Figure 9

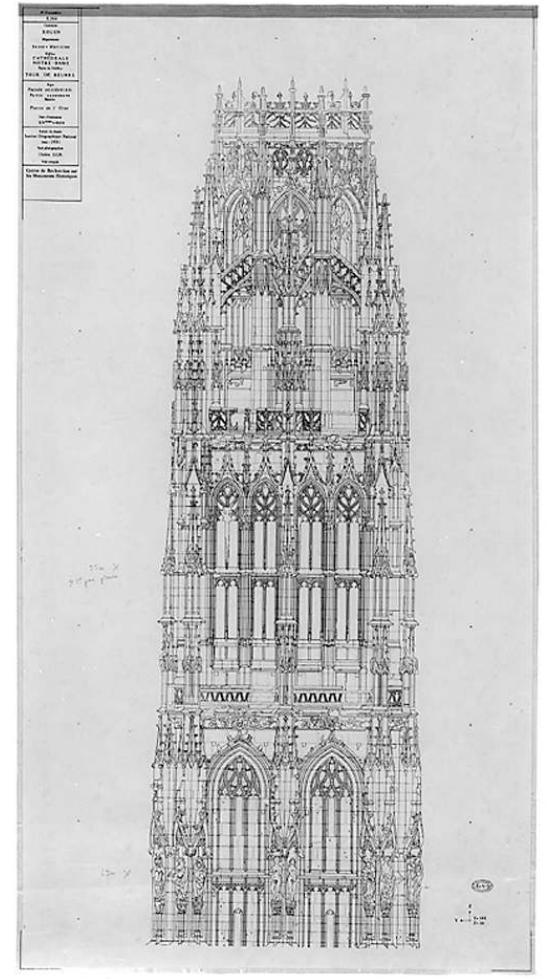

Cathédrale Notre-Dame (Rouen) : Tour de Beurre, élévation extérieure Sud, niveaux supérieurs. Relevé photogrammétrique I.G.N., 1981. Médiathèque du Patrimoine, Paris

Phot. E. Badie Modini (c) Médiathèque du patrimoine

15 A l'abbaye de Saint-Ouen, moins célèbre semble-t-il que sa sœur rivale, la Tour couronnée, plus puissante mais non moins virtuose, est le morceau de choix d'un édifice qui plusieurs fois dans l'histoire s'est trouvé placé au rang de grand monument national.

16 A l'écart du noyau primitif de la ville du haut Moyen Age, dans un bourg longtemps autonome par rapport à la commune, l'abbaye, fondée par un ministre mérovingien, a manifesté pendant dix siècles la puissance, la splendeur et la continuité spirituelle et intellectuelle de l'ordre bénédictin, soutenu aux diverses époques par le pouvoir royal. L'abbatiale est aujourd'hui et a probablement toujours été le plus vaste édifice de la ville. L'église actuelle développe le parti imposant adopté au début du XIV ${ }^{\mathrm{e}}$ siècle, réalisé et conservé au choeur dans le style gothique rayonnant le mieux maîtrisé. Ce parti a été suivi pendant près de deux siècles, non sans laisser place aux évolutions stylistiques, dans la continuité des volumes, des équilibres et des lignes maîtresses du projet. 


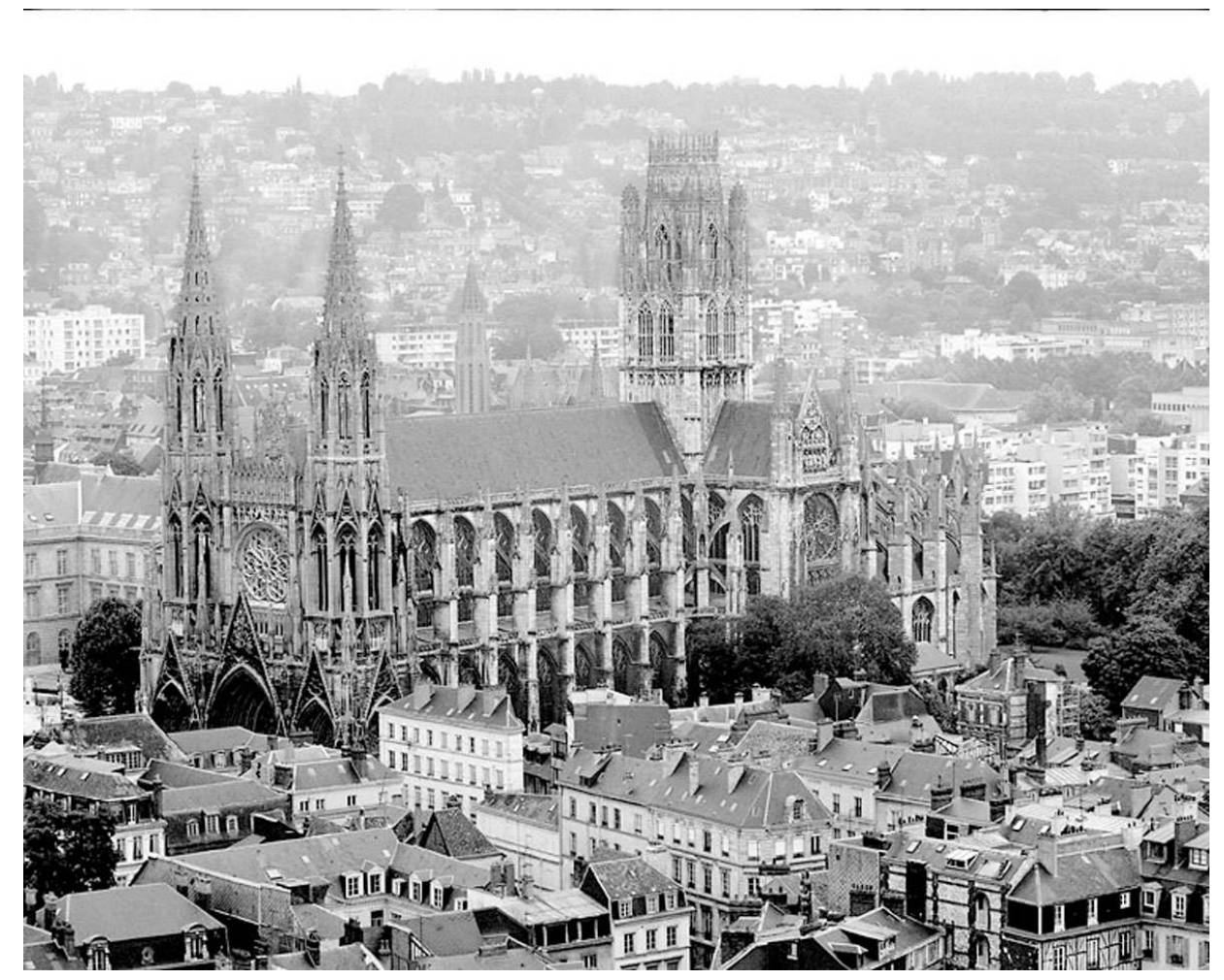

Abbaye de bénédictins Saint-Ouen (Rouen) : abbatiale, vue générale prise du sud-ouest

Phot. Inv. C. Kollmann, (c) Inventaire général, ADAGP, 1997

Notice de la base Mérimée

La tour de Saint-Ouen, de quelques mètres plus élevée que la Tour de Beurre, s'inscrit moins précisément dans l'histoire architecturale de la ville, les archives n'ayant pas encore livré la chronologie détaillée de son édification ni les noms exacts de ses maîtres d'oeuvre. Elle est, de plus, bien intégrée à l'église toute entière, de sorte qu'on hésite à la considérer pour elle-même. On comprend facilement, à la décrire, la logique de son parti mais également les hésitations et les nombreuses phases probables de sa construction; à la fois, on admet, en la voyant depuis le chevet, qu'elle achève parfaitement l'élévation du choeur, quoique de façon anachronique, et aussi, compte tenu des projets d'élévation de la façade ouest de la nef ${ }^{5}$, on admet sans réserve qu'elle aurait pu être déclinée dans les tours de façade, comme le suggèrent les gravures du XVIIe siècle, et parfaire ainsi l'ensemble de l'église... 


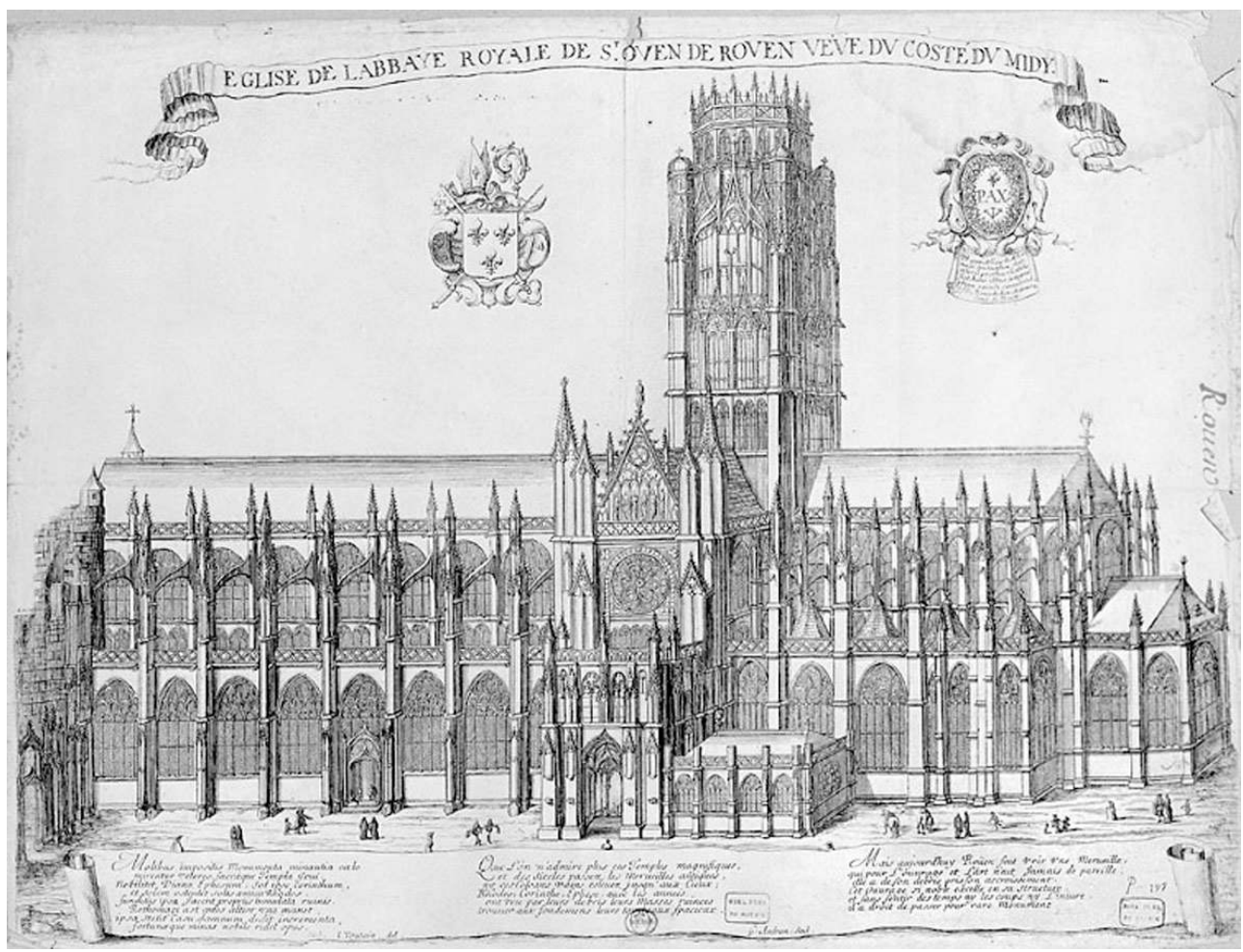

Abbaye de bénédictins Saint-Ouen (Rouen) : Elévation extérieure sud. Gravure par Audran, 1662. Rouen, Bibl. mun

Phot. Inv. Y. Miossec, (c) Inventaire général, ADAGP, 1980

Mais considérons-la pour elle-même. Sa première caractéristique est d'être une tour de croisée, ce qui dans une église dont les voûtes sont lancées à $30 \mathrm{~m}$ du sol indique la difficulté de l'entreprise. De plan carré, sur une base d'une douzaine de mètres, les quatre piliers qui la portent ont certainement été implantés et, pour les piles est au moins, élevés jusqu'aux voûtes avant la fin du XIV siècle. L'achèvement du transept date du début du XVe siècle et a du comprendre au moins le début d'édification de la tour proprement dite. 
Figure 12

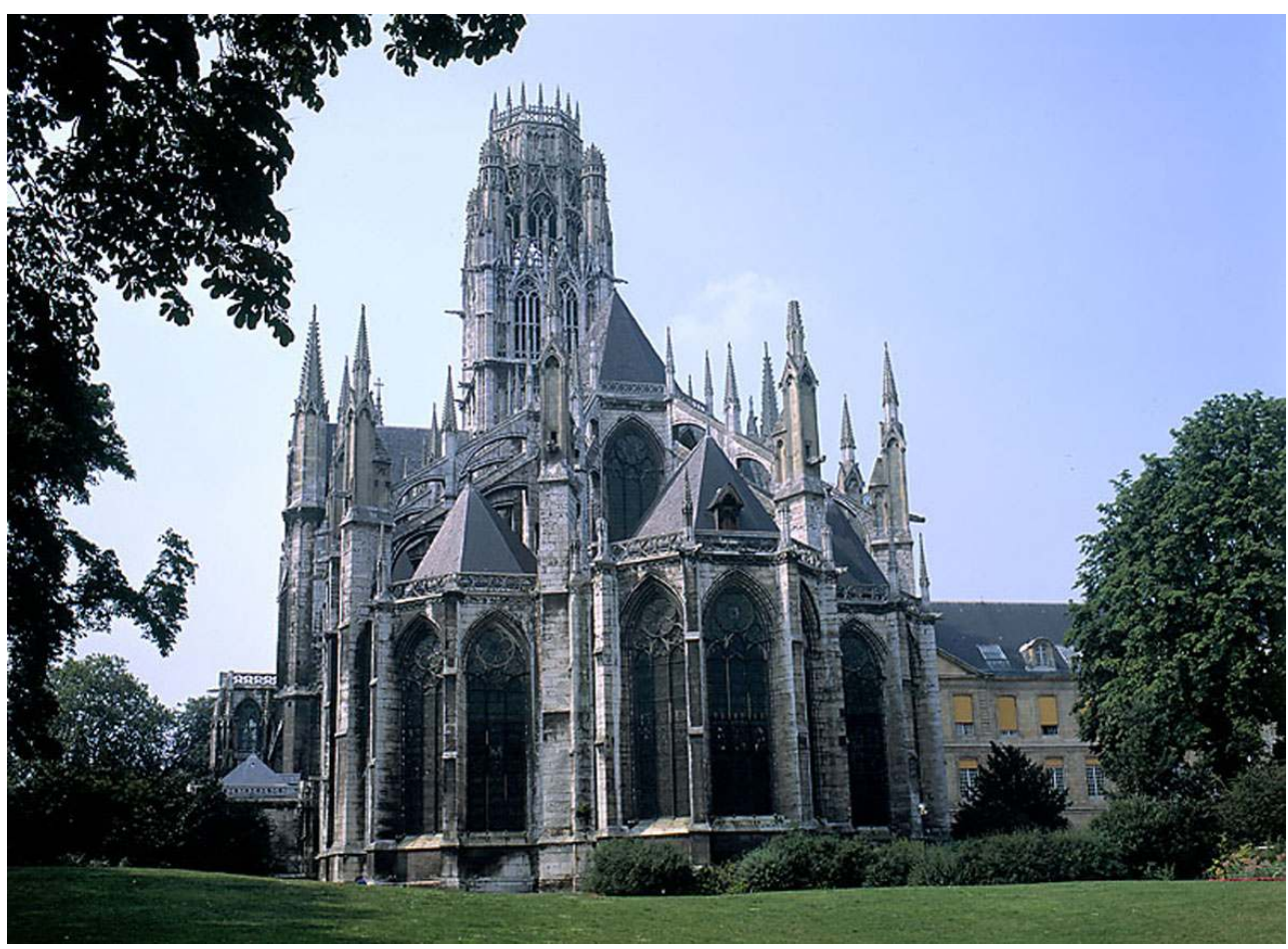

Abbaye de bénédictins Saint-Ouen (Rouen) : abbatiale, chevet, vue générale

Phot. Inv. D. Couchaux, (c) Inventaire général, ADAGP, 1995 
Figure 13

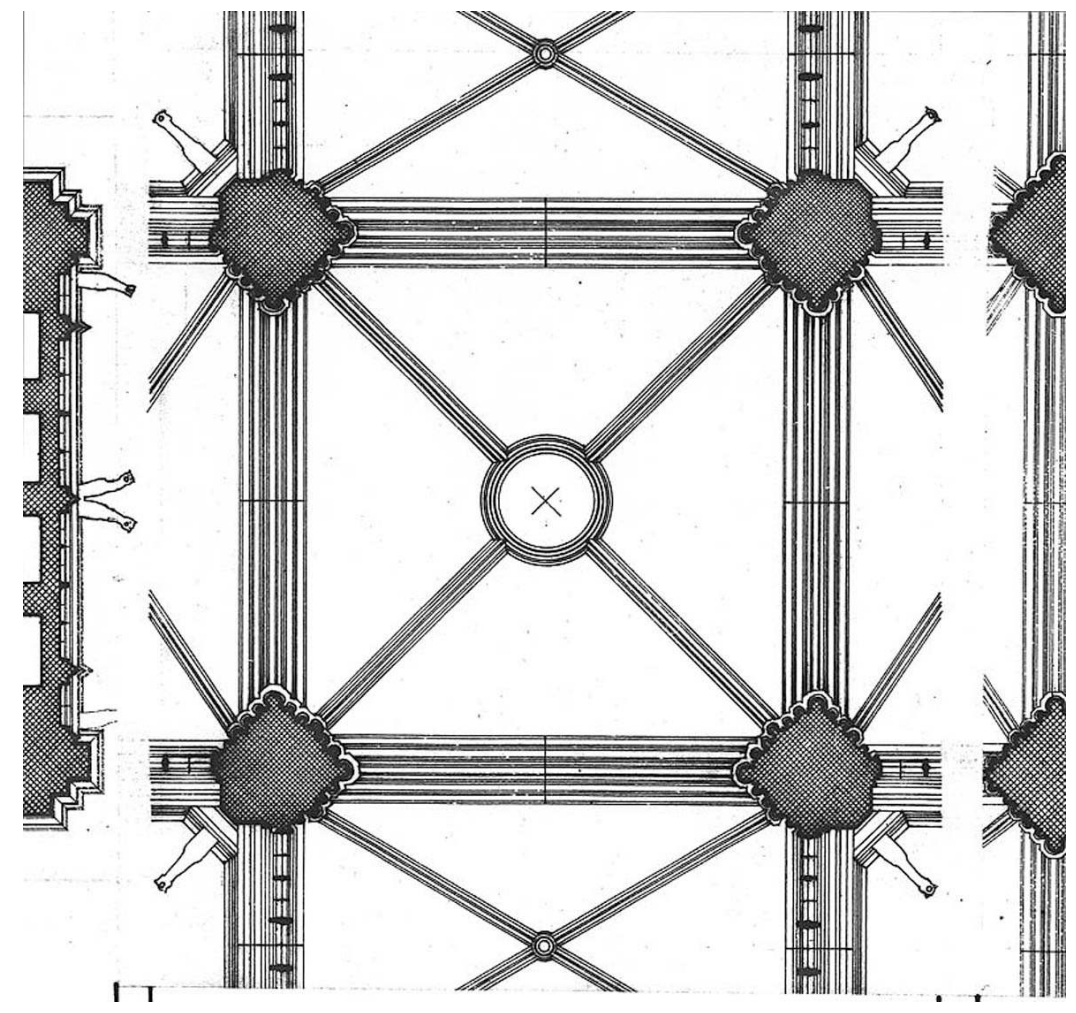

Abbaye de bénédictins Saint-Ouen (Rouen) : abbatiale, tour du transept, plan avec projection de la voûte de la nef. Relevé D. Moufle, 2000

Phot. Inv. P. Fortin, (c) Inventaire général, ADAGP 
Figure 14

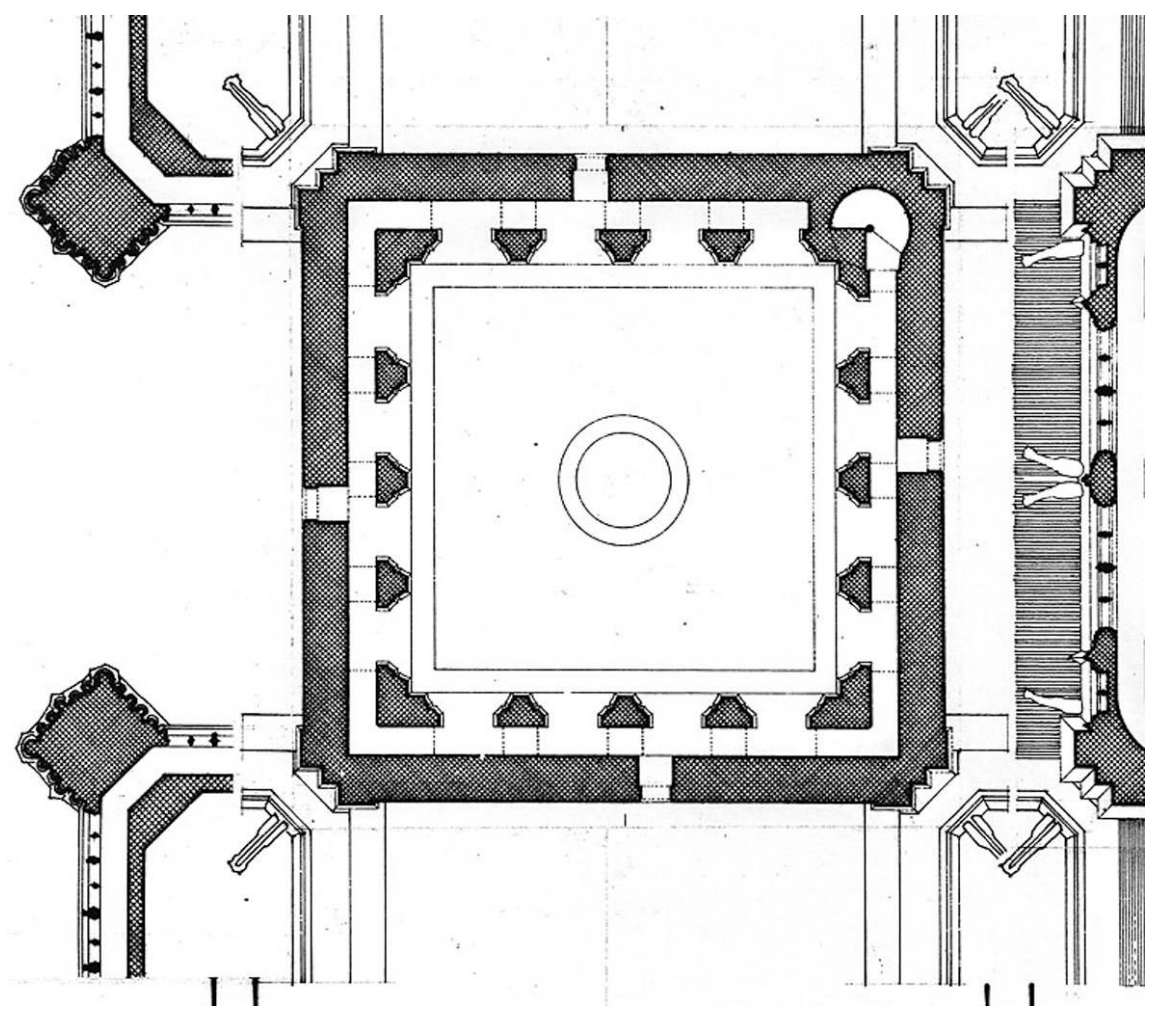

Abbaye de bénédictins Saint-Ouen (Rouen) : abbatiale, tour du transept, plan au niveau de la base du beffroi. Relevé D. Moufle, 2000

Phot. Inv. P. Fortin, (c) Inventaire général, ADAG 


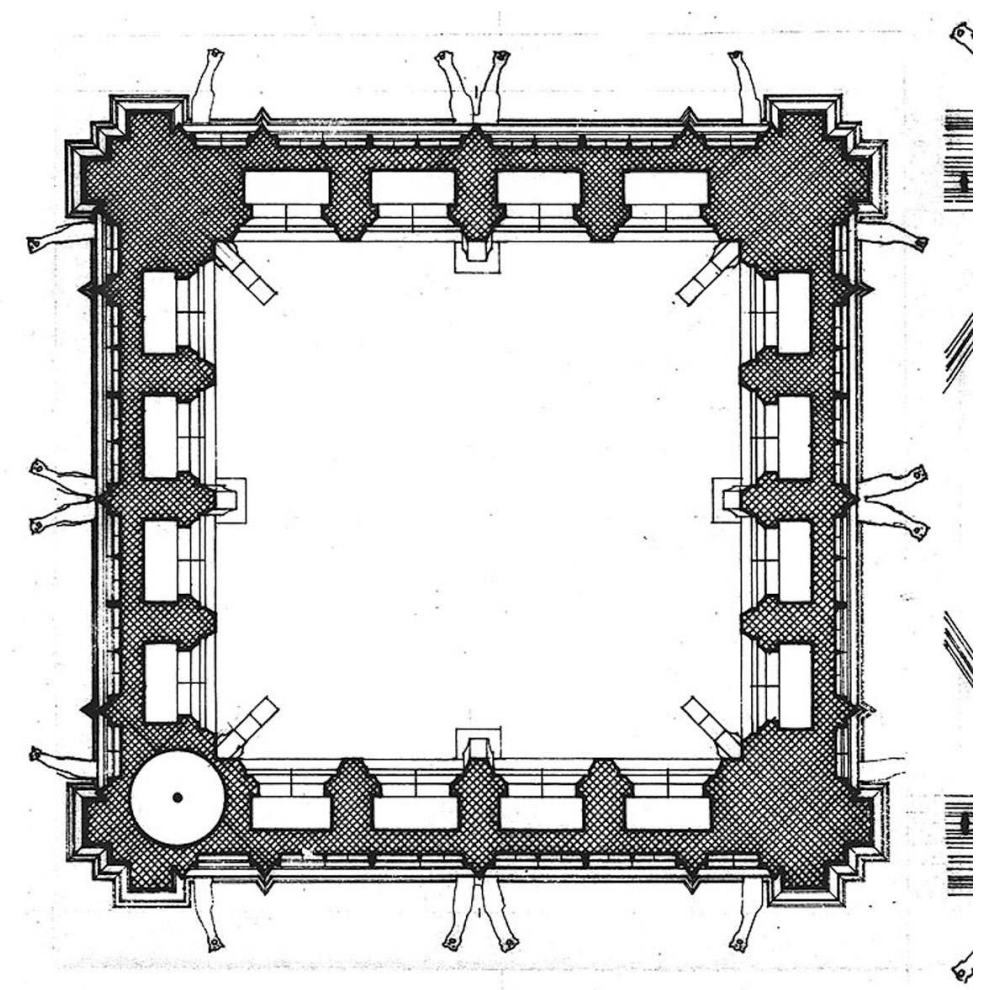

Abbaye de bénédictins Saint-Ouen (Rouen) : abbatiale, tour du transept, plan au niveau du beffroi. Relevé D. Moufle, 2000

Phot. Inv. P. Fortin, (c) Inventaire général, ADAGP

19 L'histoire a retenu les difficultés des maçons devant le bouclage des piliers observé à ce moment de la construction, en $1441^{6}$. Les croisées de transept ont toujours été le point névralgique des grands édifices à vaisseaux, concentrant sur ce grand vide central l'essentiel des poussées architectoniques. 
Figure 16

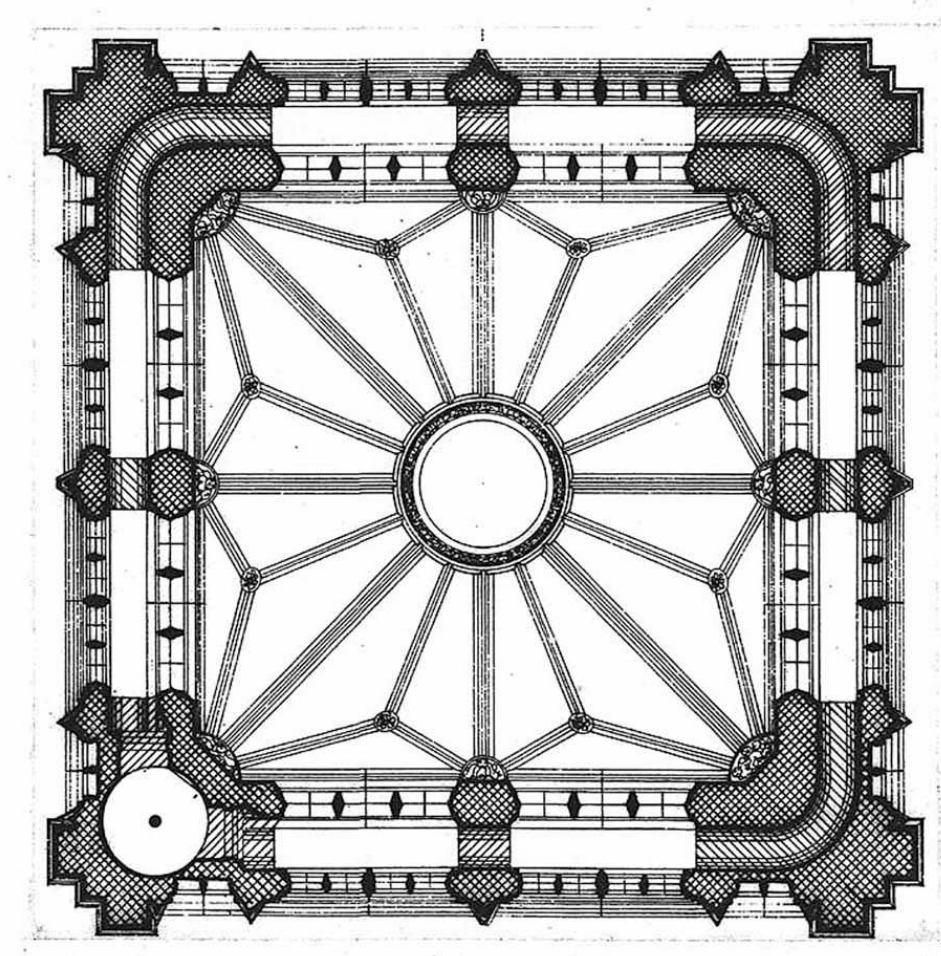

Abbaye de bénédictins Saint-Ouen (Rouen) : abbatiale, tour du transept, plan au niveau des fenêtres hautes avec projection de la voûte. Relevé D. Moufle, 2000

Phot. Inv. P. Fortin, (c) Inventaire général, ADAGP 
Figure 17

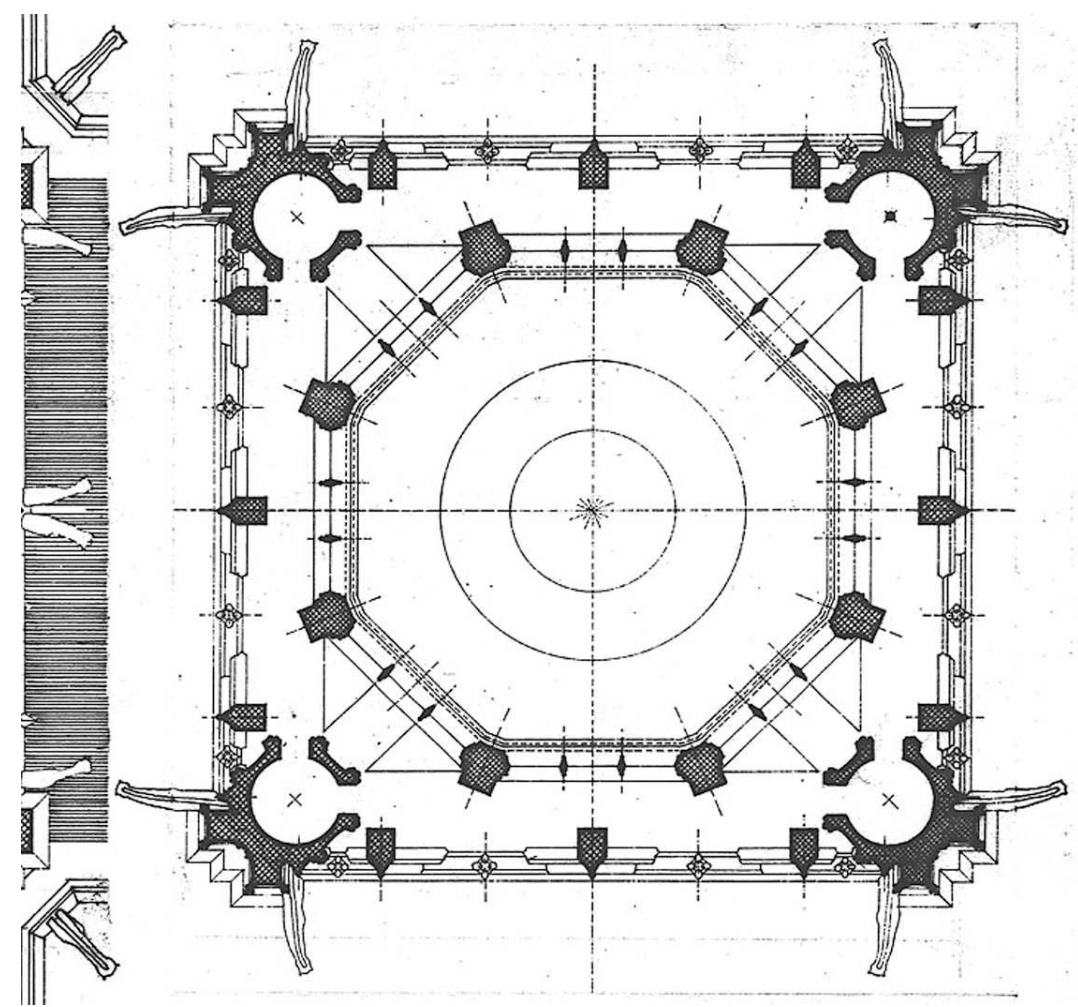

Abbaye de bénédictins Saint-Ouen (Rouen) : abbatiale, tour du transept, plan au niveau des baies de la couronne. Relevé D. Moufle, 2000

Phot. Inv. P. Fortin, (c) Inventaire général, ADAGP 


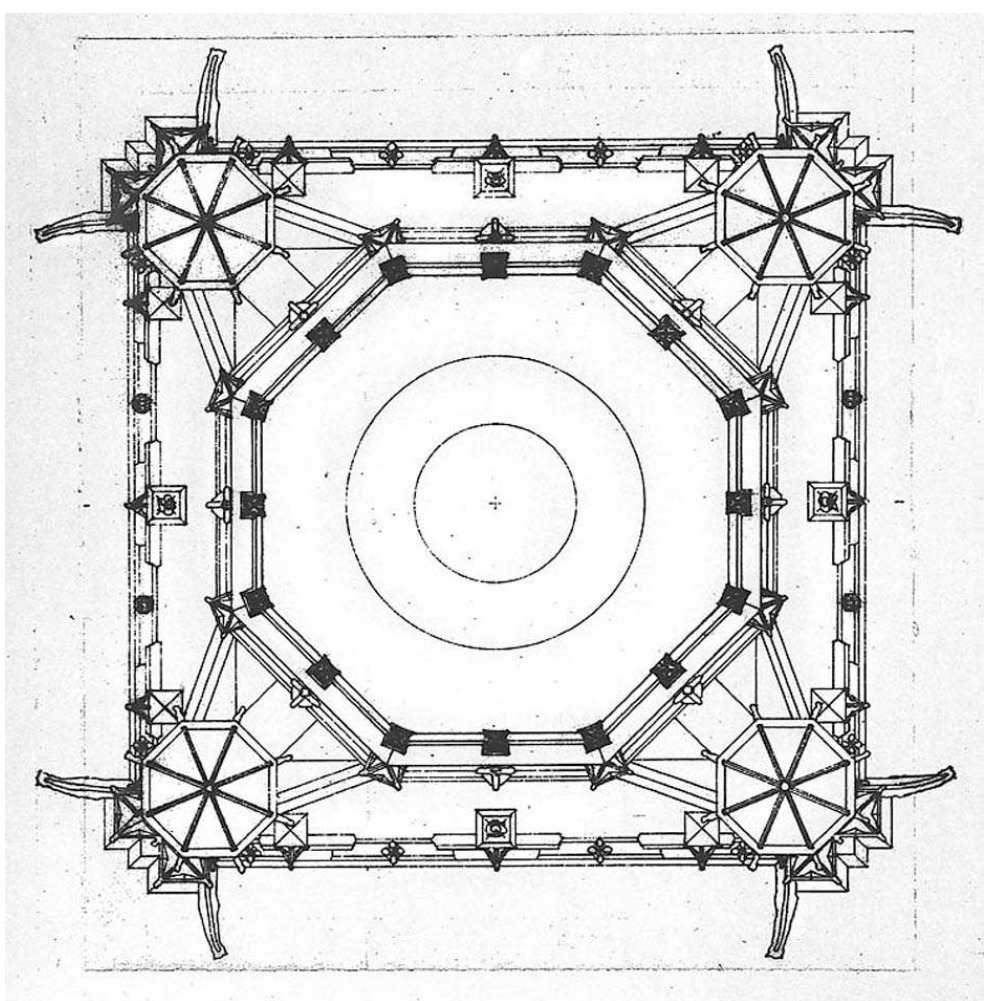

Abbaye de bénédictins Saint-Ouen (Rouen) : abbatiale, tour du transept, plan au niveau du faîtage de la couronne. Relevé D. Moufle, 2000

Phot. Inv. P. Fortin, (c) Inventaire général, ADAGP

Le parti traditionnel en Normandie de placer là une lanterne, s'il est remarquable pour la distribution de l'espace et la répartition de la lumière, est aussi parfois déraisonnablement ambitieux. Un tel projet a semble-t-il été envisagé et peut-être partiellement réalisé à Saint-Ouen où, au-dessus de la voûte simple sur croisée d'ogives, on est tenté de voir, autour de l'actuel beffroi, la structure d'une lanterne. Les murs des quatre faces présentent en effet un dédoublement et un jeu de percements tout à fait comparables aux grandes lanternes gothiques normandes. Le haut volume central est couvert d'une voûte d'ogives à huit branches avec clef annulaire ${ }^{7}$ que l'on imaginerait bien, étant donné la qualité de leur décor, devoir être visibles depuis le sol du transept. 


\section{Figure 19}

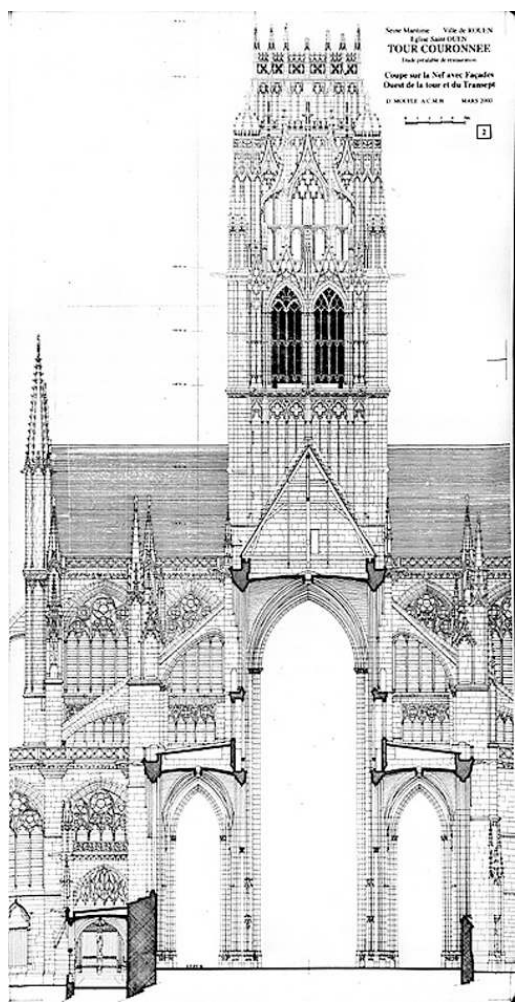

Abbaye de bénédictins Saint-Ouen (Rouen) : abbatiale, tour du transept, plan au niveau du faîtage de la couronne. Relevé D. Moufle, 2000

Phot. Inv. P. Fortin, (c) Inventaire général, ADAGP 


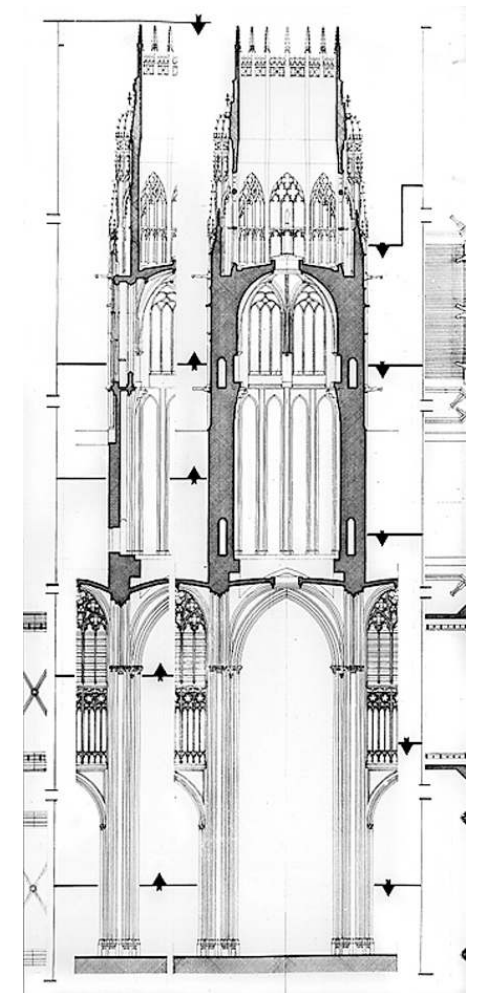

Abbaye de bénédictins Saint-Ouen (Rouen) : abbatiale, tour du transept, plan au niveau du faîtage de la couronne. Relevé D. Moufle, 2000

Phot. Inv. P. Fortin, (c) Inventaire général, ADAGP

A quel moment un tel changement de programme a-t-il pu intervenir? A défaut de pouvoir suivre avec les archives le détail des financements, le déroulement du chantier et le nom des maîtres d'oeuvre, force est de s'en tenir aux hypothèses et aux données contemporaines de cette construction manifestement achevée avant 1524 puisqu'elle figure entière sur le Livre des Fontaines. Le réseau fleurdelysé des fenêtres du second niveau extérieur affirme avec vigueur les liens avec la monarchie, celle de Louis XI ou de Charles VII, et indique une campagne soutenue par la royauté française et postérieure à la victoire de Formigny (1450). Au-dessus de ce niveau, le changement de matériau de construction, opposant au calcaire à silex des carrières de Seine le calcaire ocre (Vergelé) des carrières de l'Oise, semble bien indiquer un changement de maitrise d'oeuvre, si ce n'est de maîtrise d'ouvrage que l'on serait tenté de faire coïncider avec l'arrivée, en 1485, du tout-puissant et fortuné abbé Antoine Bohier, réputé, dans l'histoire de l'abbaye, comme bâtisseur. En attendant les dépouillements d'archives qui peut-être confirmeront ces propositions, il est raisonnable aujourd'hui d'envisager l'articulation de la construction de ces parties hautes en deux phases : celle d'une tour lanterne, entre 1440 et 1480 , dont l'ouverture sur la nef aurait finalement été bouchée par une voûte sur croisée d'ogives pour stabiliser les poussées des vaisseaux de nef et de transept, toujours en chantier à cette époque; puis entre 1490 et 1510 environ, construction du couronnement sur l'assise carrée du beffroi et dans le prolongement des batteries de contreforts d'angle. La rupture de parti est évidente entre ces deux campagnes, et pourtant, l'habileté des architectes a parfaitement maîtrisé la transition tout en introduisant des innovations particulièrement séduisantes. 


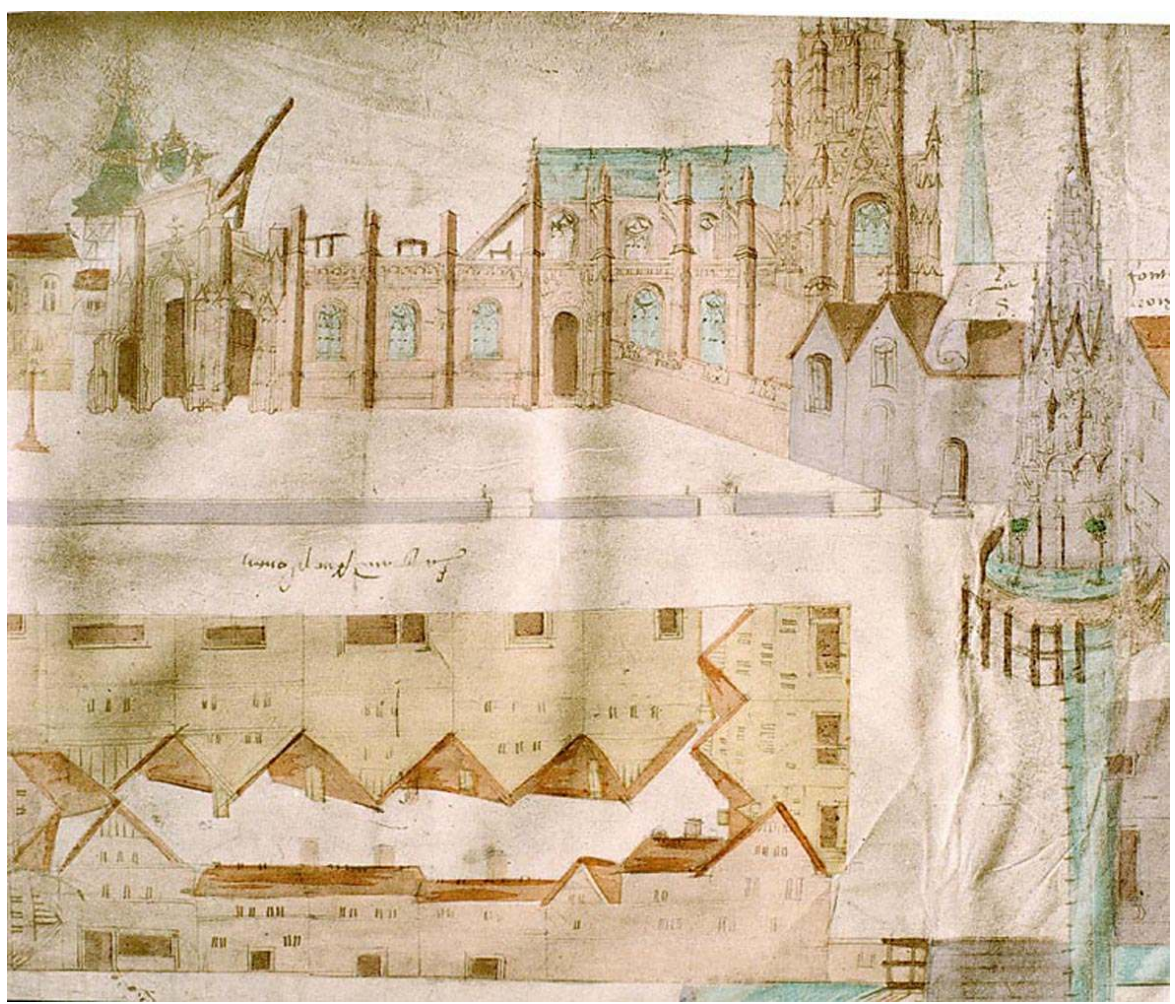

Abbaye de bénédictins Saint-Ouen (Rouen) : Abbatiale, chantier de la nef. Aquarelle, 1524. Livre des Fontaines, reproduction fac-similé par Victor Sanson, Rouen, 1911

Phot. Inv. Y. Miossec, (c) Inventaire général, ADAGP, 1992

Au niveau des rampants de toit des vaisseaux, la base de la tour est aveugle. La masse de pierre est très adroitement habillée par une arcature fine qui rythme chaque face, selon les travées du niveau supérieur, par des réseaux très délicats, contrastant avec la robuste corniche feuillagée. Là commencent les parties hautes où s'étagent une démultiplication d'organes et un allègement des volumes : tout d'abord, sur un plan toujours carré, une ordonnance large de grandes baies jumelées encadrée de puissants piliers à contreforts jumelés. Les huit grandes fenêtres de ce niveau, par la richesse de leur remplage, par les réseaux fleurdelysés, par les couronnements d'arc en gâbles évidés à soufflets, affirment une respiration puissante, une perméabilité des espaces, soutenues de façon contrastée par la vigueur des contreforts montant nus vers l'étage supérieur. 


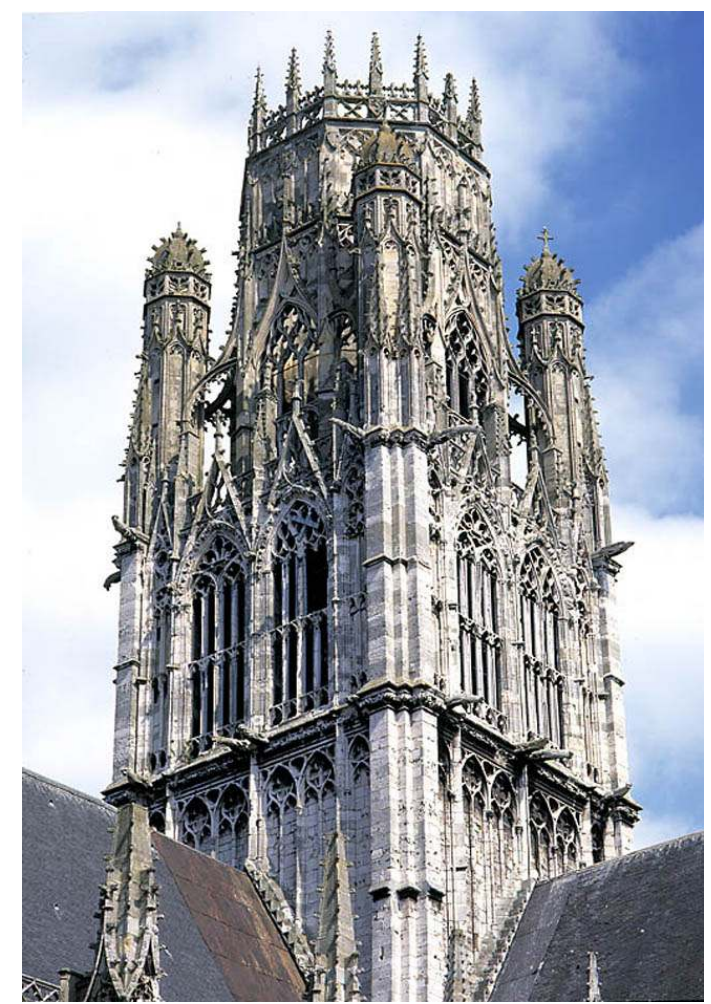

Abbaye de bénédictins Saint-Ouen (Rouen) : Abbatiale, tour du transept, vue générale prise du sudouest

Phot. Inv. D. Couchaux, (c) Inventaire général, ADAGP, 1997

Sur la terrasse carrée couvrant ce niveau, l'architecte pose une structure complètement découverte et aérienne formant comme un portique octogonal dont l'amortissement en frise dessine la couronne. Le changement de matériau, le travail de stéréotomie et les détails d'ornementation révèlent un véritable changement de parti, mais la liaison est très habile et l'architecte paraît parfaitement poursuivre l'oeuvre engagée par ses prédécesseurs.

On peut observer cette transition dans les quatre tourelles d'angle qui sont une caractéristique majeure de cette tour. Elles jouent secondairement le rôle de culée d'arcboutant. De plan octogonal, leurs faces externes sont au nu des contreforts d'angle, reliées par des pinacles. Ces tourelles aveugles et vides sont amorties par une fausse petite balustrade à fleurons et couvertes de petites coupoles à huit pans sommées de croix de pierre. Cette disposition est tout à fait originale et, à Rouen, c'est véritablement une première que de placer et d'afficher ainsi quatre coupoles sphériques en couvrement de ces volumes centrés. 


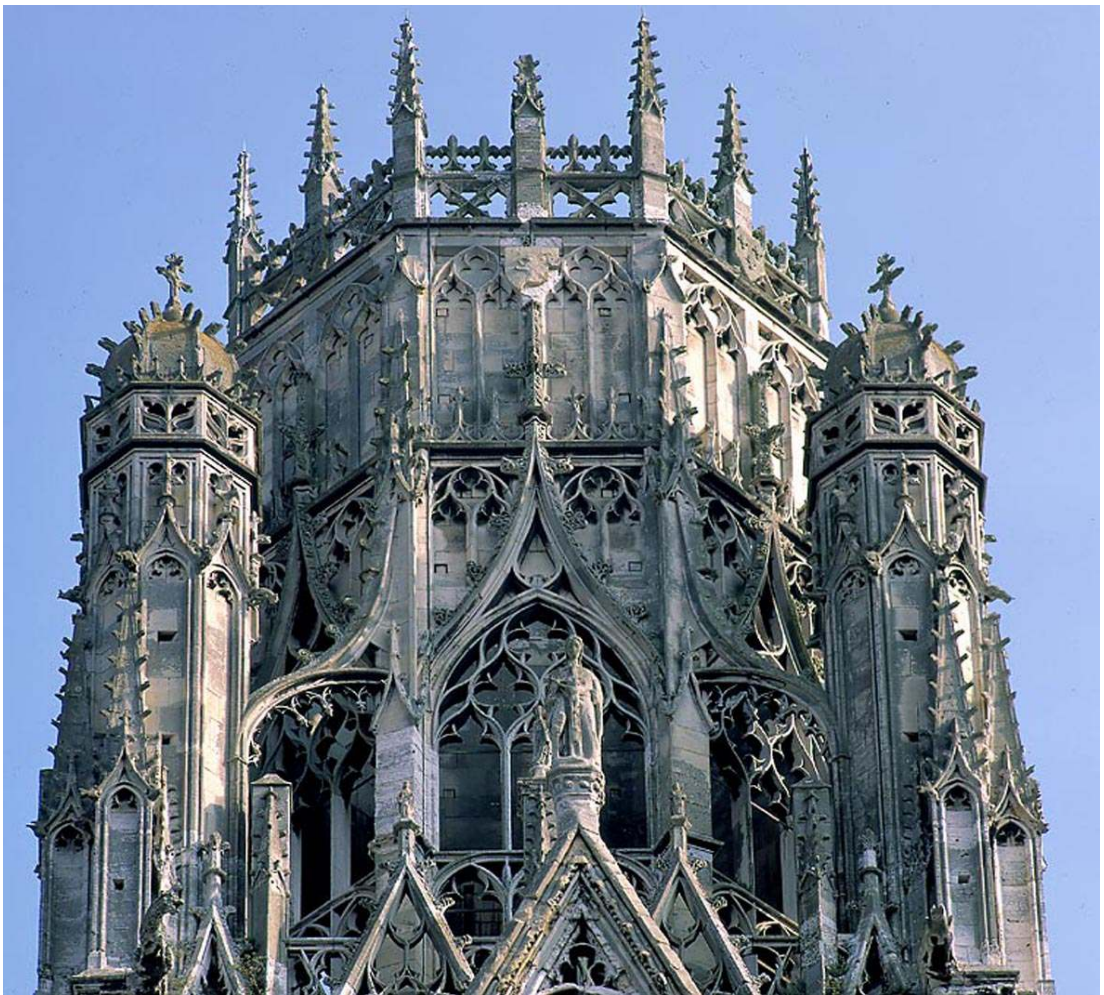

Abbaye de bénédictins Saint-Ouen (Rouen) : Abbatiale, tour du transept, couronnement Phot. Inv. D. Couchaux, (c) Inventaire général, ADAGP, 1995

Ces quatre organes d'angle, robustes et quelque peu massifs, encadrent la tour centrale, entièrement ouverte sur ses huit faces par des baies libres à triple meneaux et réseaux. Les huit supports, épaulés par des contreforts à feston, portent une simple enveloppe maçonnée, au parement intérieur lisse, sans coursière ni couvrement d'aucune sorte. Cette enveloppe est chargée à l'extérieur d'un décor très géométrique d'arcatures aveugles, de pinacles et de corniches rappelant les niveaux inférieurs.

De l'ensemble, effectivement très vide, ressort une transparence extraordinaire : vu du pied de la tour, le ciel passe au travers des maçonneries, et à distance, le regard passe véritablement de part en part de la tour. 
Figure 24

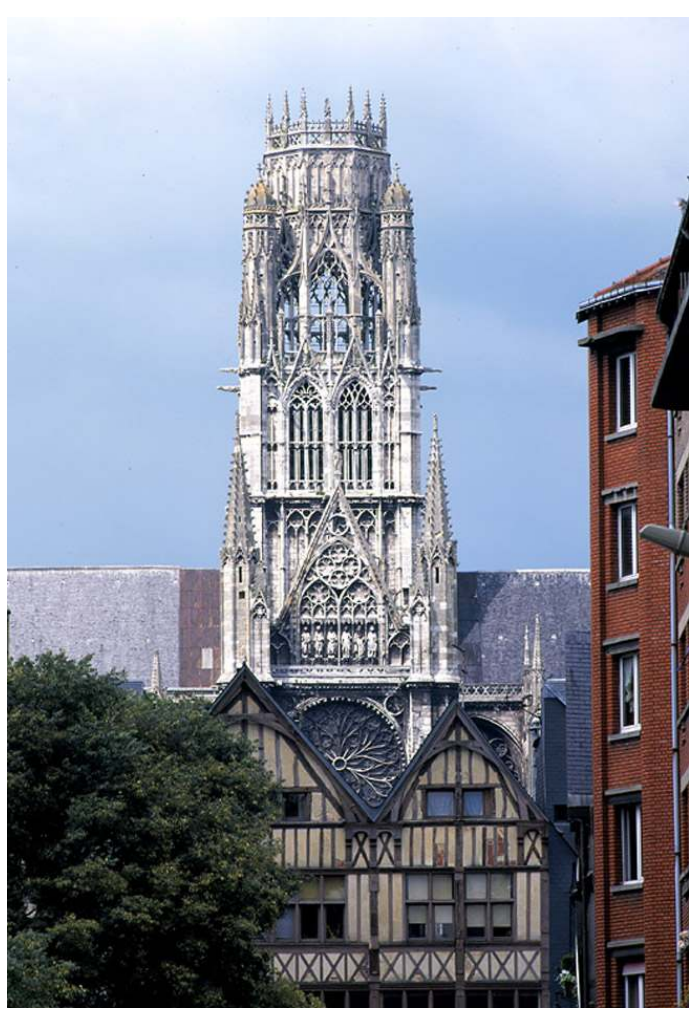

Abbaye de bénédictins Saint-Ouen (Rouen) : abbatiale, tour du transept, vue générale prise du sud Phot. Inv. D. Couchaux, (c) Inventaire général, ADAGP, 1997

Sans connaître encore la genèse de cette création un peu fantastique, force est de la lier aux commandes du dispendieux Antoine Bohier et de la situer dans le double contexte des audaces les plus folles du gothique flamboyant à son terme, mais aussi à l'introduction d'un art importé d'Italie sans lequel on imaginerait mal les quatre petites coupoles de Saint-Ouen.

Pierre de Valence ${ }^{8}$, architecte du Val de Loire travaillant à Saint-Gatien de Tours en 1500, était à Rouen au moment de l'édification de cette tour. Il est appelé vers 1503 par Georges d'Amboise sur le chantier de Gaillon et réside à Rouen où il suit la pose de céramiques à la galerie de l'archevêché. Manifestement très proche du cardinal, il semble apprécié pour sa connaissance de l'architecture ultramontaine qu'il sait mettre en oeuvre dans des réalisations luxueuses : sculpture, pavements, fontaines... Il participe à la conférence de 1504 devant le chapitre de la cathédrale pour décider si la Tour de Beurre devait être achevée « à aiguille ou à terrasse ». On le voit très actif à Gaillon, en 1506, où il dirige les travaux de sculpture à la galerie du jardin et à la chapelle. C'est lui qui met en place la fontaine de Gaillon en 1508 et, la même année, à Saint-Ouen, il aménage également une fontaine. On est tenté à Rouen de voir en cet artiste, qui retourne définitivement à Tours en 1510, un décorateur ou un architecte de second oeuvre, apprécié pour sa culture ultramontaine. Or c'est à lui que l'on attribue ${ }^{9}$ l'achèvement de la tour nord de SaintGatien de Tours qu'il est intéressant de comparer avec Saint-Ouen. Sur la base carrée des anciennes tours romanes de façade, entièrement rhabillées à la fin $d u \mathrm{XV}^{\mathrm{e}}$ siècle d'un décor flamboyant, le chapitre demande en 1507 à Pierre de Valence de placer un couronnement octogonal fermé dont le couvrement est une véritable coupole avec 
lanternon formant belvédère. Au moment où s'achevait le chantier de la chapelle haute de Gaillon, disparue et encore assez mal connue mais dont on peut se demander si la tour centrale n'était pas couronnée d'un lanternon en dôme, alors qu'il avait pu suivre le chantier de Saint-Ouen, l'oeuvre de Tours, loin de la tradition des flèches gothiques du Nord, établit une coupole octogonale dont l'analyse montrerait les nombreux repentirs et la fragilité (elle nécessite un jeu de renforts et de tirants de charpente), mais dont l'équilibre est proche de la couronne de Saint-Ouen.

Figure 25

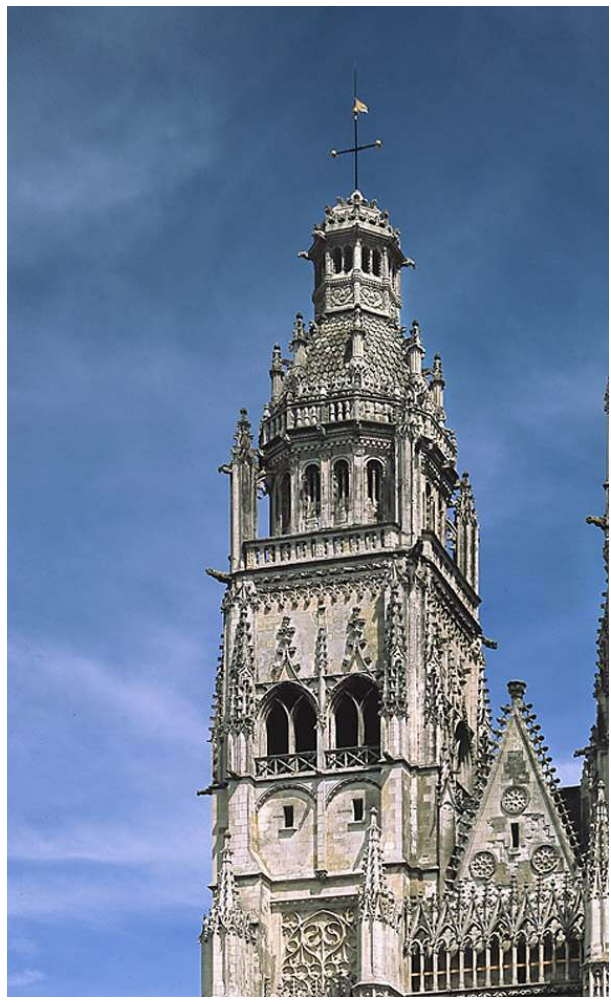

Cathédrale Saint-Gatien (Tours) : Tour Nord de la façade ouest, vue générale Phot. Inv. R. Malnoury, (c) Inventaire général, ADAGP, 2001

Notice de la base Mérimée

Placées comme les pièces maitresses d'un jeu d'échecs, les deux tours de Rouen se renvoient, comme des rivales, des silhouettes semblables au premier abord et que l'analyse distingue avec un certain plaisir. La Tour de Beurre incarne, dans toute sa dignité, la forte tradition des architectes du chapitre entièrement dévoués au style gothique flamboyant. Leur habileté est d'avoir su dépasser leur projet contrarié de flèche en une couronne (était-ce leur invention?) qui achève leur chantier avec la plus grande adresse et une raisonnable économie. La couronne de Saint-Ouen, plus généreuse et plus aérienne encore, est un ouvrage un peu artificiellement posé au sommet de l'édifice, mais qui, parfaitement dégagé et proportionné, comme la couronne d'un monarque, affiche à la fois richesse et pouvoir.

Les deux tours de Rouen semblent avoir fortement frappé l'imaginaire des architectes bâtisseurs d'églises de leur temps, mais aussi de ceux des siècles suivants... Sans pouvoir toujours bien déceler laquelle, de la Tour de Beurre ou de la Tour couronnée, a servi de modèle, la forme générale si caractéristique et surtout l'amortissement en couronne ont 
établi l'image originale de tours flamboyantes bien achevées, compromis entre la terrasse, trop inaboutie (la Tour Saint-Jacques de Paris) que ne pourra accepter Viollet-leDuc et la flèche, ambitieuse mais coûteuse, fragile et finalement quelquefois encombrante comme à la cathédrale de Cologne, ou inconséquente, comme les flèches placées par Grégoire, en 1842, à la façade de l'abbaye de Saint-Ouen.

\section{Figure 26}

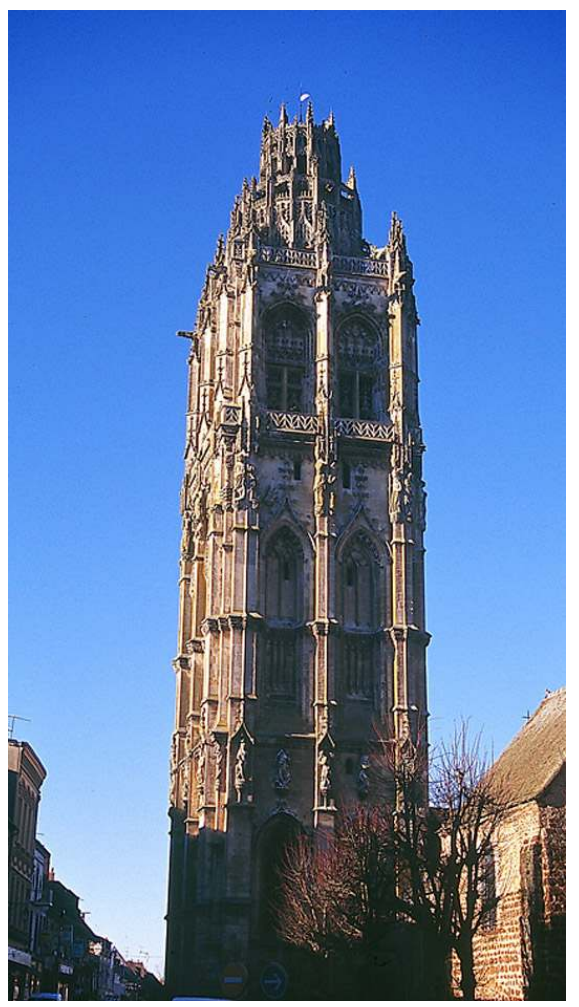

Eglise paroissiale Sainte-Marie-Madeleine (Verneuil-sur-Avre) : Tour-clocher, élévation extérieure est, vue générale prise du sud-est

Phot. Inv. F. Verdier, (c) Inventaire général, ADAGP, 2001

On peut ainsi suivre, de la tour de la Madeleine de Verneuil-sur-Avre (Eure) à la Tribune Tower de Chicago, toute une série de chefs-d'oeuvre dont la filiation avec Saint-Ouen ou avec la cathédrale est plus ou moins évidente.

Notice de la base Mérimée 


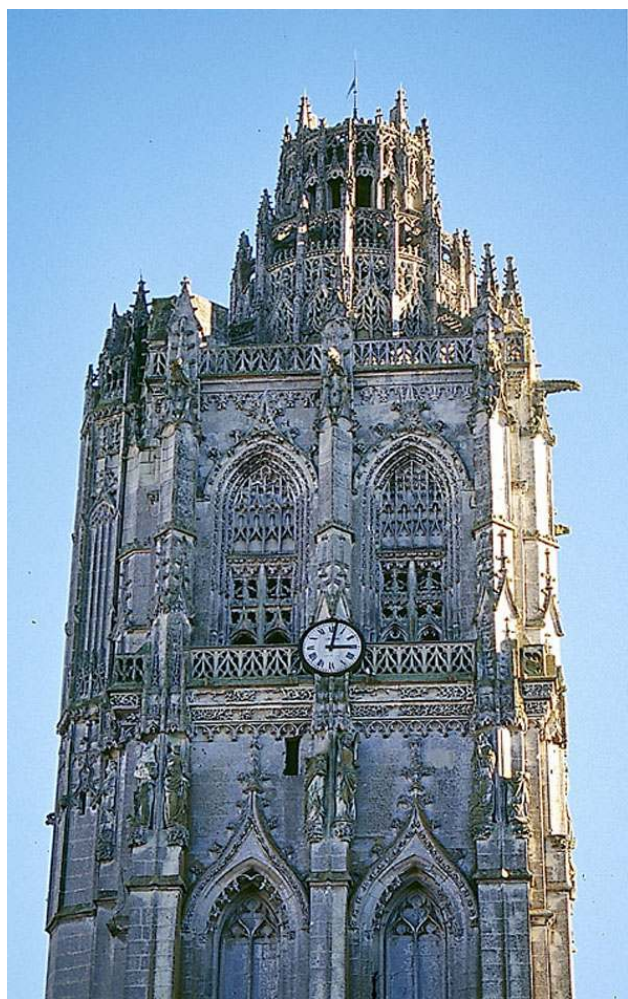

Eglise paroissiale Sainte-Marie-Madeleine (Verneuil-sur-Avre) : Tour-clocher, élévation extérieure ouest, niveaux supérieurs

Phot. Inv. F. Verdier, (c) Inventaire général, ADAGP, 2001

Par les proportions, la position et l'autonomie du projet, c'est peut-être à la Tour de Beurre que se réfère la tour de la Madeleine de Verneuil. Arthus Filion en fit achever la construction, vers 1520 , avant de devenir évêque de Senlis ${ }^{10}$. On ne sait pas le nom de l'architecte de cette magnifique tour de $55 \mathrm{~m}$ de haut, mais il ne fait pas de doute que celui-ci connaissait bien celles de Rouen.

Notice de la base Mérimée 
Figure 28

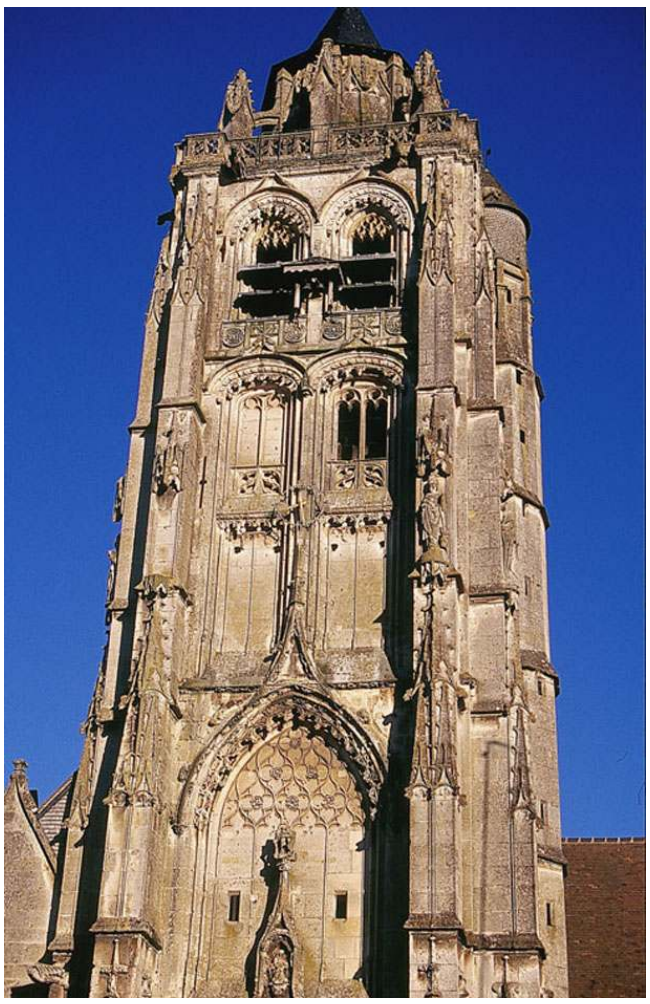

Eglise paroissiale Saint-Germain (Rugles) : Tour-clocher, élévation extérieure sud, niveaux supérieurs, vue prise du sud-est

Phot. Inv. F. Verdier, (c) Inventaire général, ADAGP, 2001 


\section{Figure 29}

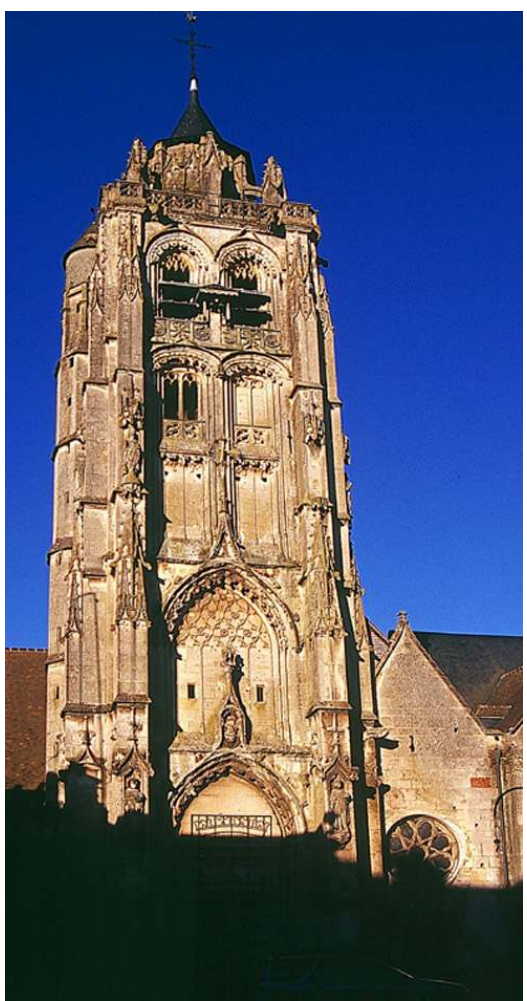

Eglise paroissiale Saint-Germain (Rugles) : Tour-clocher, élévation extérieure sud, vue prise du sud Phot. Inv. F. Verdier, (c) Inventaire général, ADAGP, 2001

33 Beaucoup plus modeste, mais suivant le même modèle, la tour de Rugles (Eure), à quelques kilomètres de Verneuil, manifeste un même engouement pour cette forme architecturale. 
Figure 30

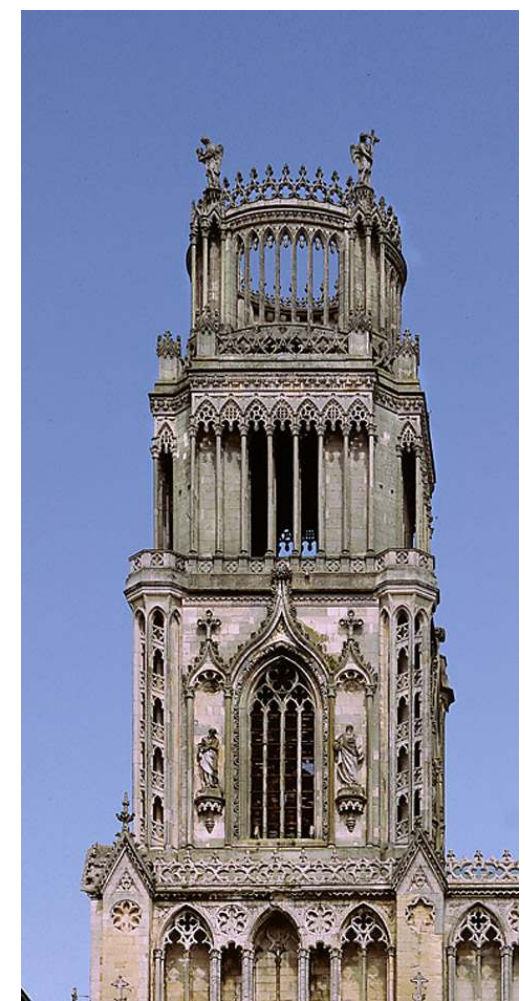

Cathédrale Sainte-Croix (Orléans) : Tour Nord de la façade ouest, vue générale Phot. Inv. R. Malnoury, (c) Inventaire général, ADAGP, 1997

Lorsqu'il fallut, près de deux siècles plus tard, achever la cathédrale d'Orléans, LouisFrançois Trouard, à partir de 1765, et surtout Pierre-Adrien Pâris, à partir de 1787, ont agi en véritables archéologues en s'inspirant de plusieurs édifices réputés: Strasbourg, Beauvais, Amiens, Dijon et Bourges. Pour les tours de façade, ils ont fait relever le couronnement de Saint-Ouen qui a été directement copié dans les projets de Trouard (plan octogonal). C'est Pâris, qui au moment de la mise en oeuvre, entre 1787 et 1790, a préféré le plan circulaire, mais le résultat ne cache pas cette filiation ${ }^{11}$.

Notice de la base Mérimée 


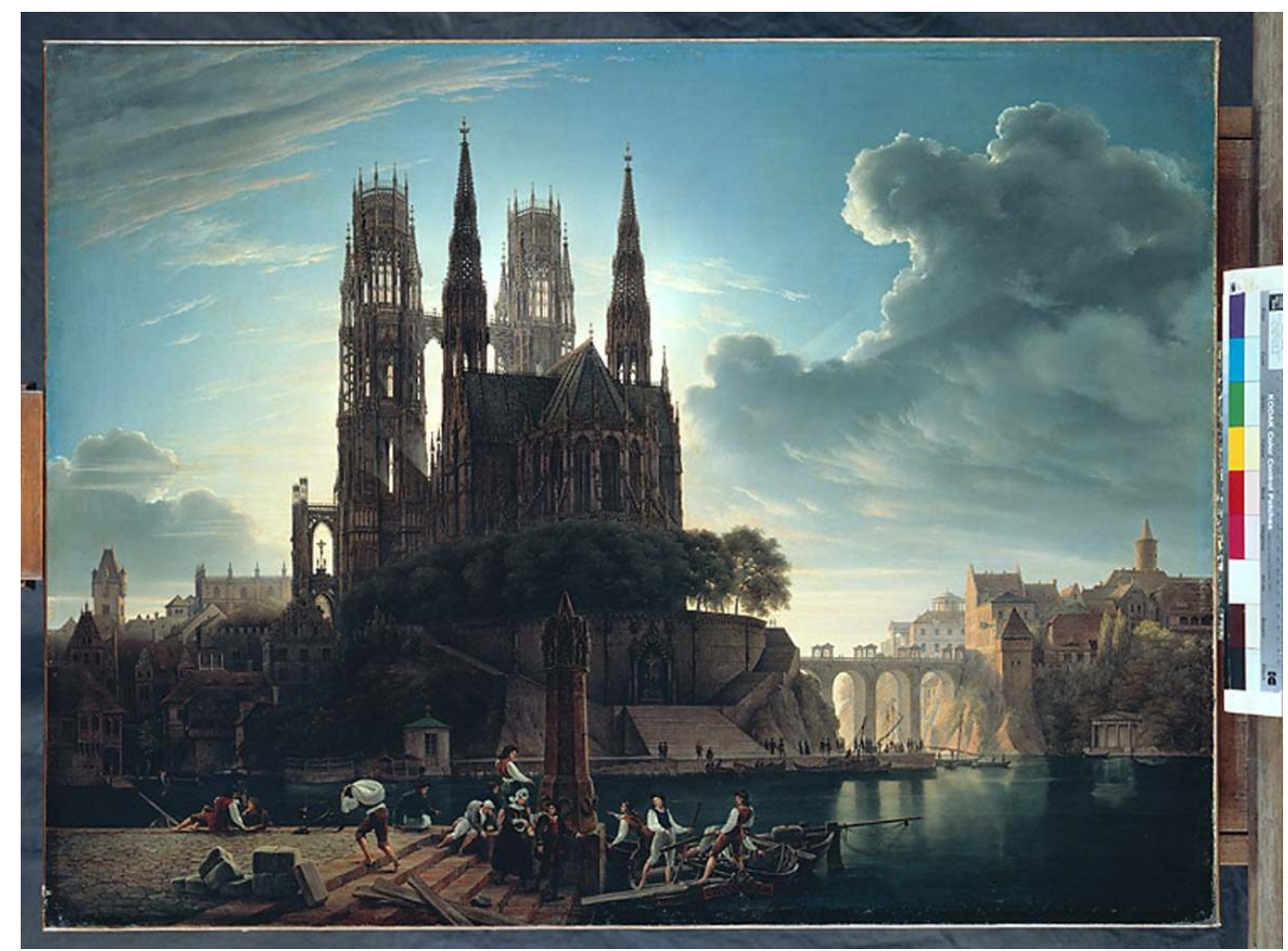

Tableau : cathédrale gothique. Huile sur toile par Wilhelm Ahlborn d'après Karl Friedrich Schinkel. (c) Nationalgalerie Berlin

Lorsqu'il conçoit un modèle de cathédrale gothique pour le diorama de Berlin, Karl Friedrich Schinkel, en 1827, propose un vaste édifice hybride copié d'une de ses toiles romantiques peinte en 1813 (aujourd'hui détruite mais connue par une copie par Ahlborn de 1825). L'édifice est assez disproportionné et l'archéologue peut y déceler de nombreuses références aux grands monuments du gothique français assemblées avec nombre d'anachronismes et d'incohérences. Le tableau fait cependant une très forte impression, due en particulier à l'effet de contre-jour et à la légèreté magique des volumes bien observée sur les édifices flamboyants, en particulier dans les deux tours de façade, toute en transparence, doubles reproductions de celles de Saint-Ouen, corrigées seulement au niveau des pinacles des tourelles d'angle que Schinkel, puriste avant les rationalistes français du milieu du siècle, préfère aux petits dômes si caractéristiques de la Tour couronnée. 
Figure 32

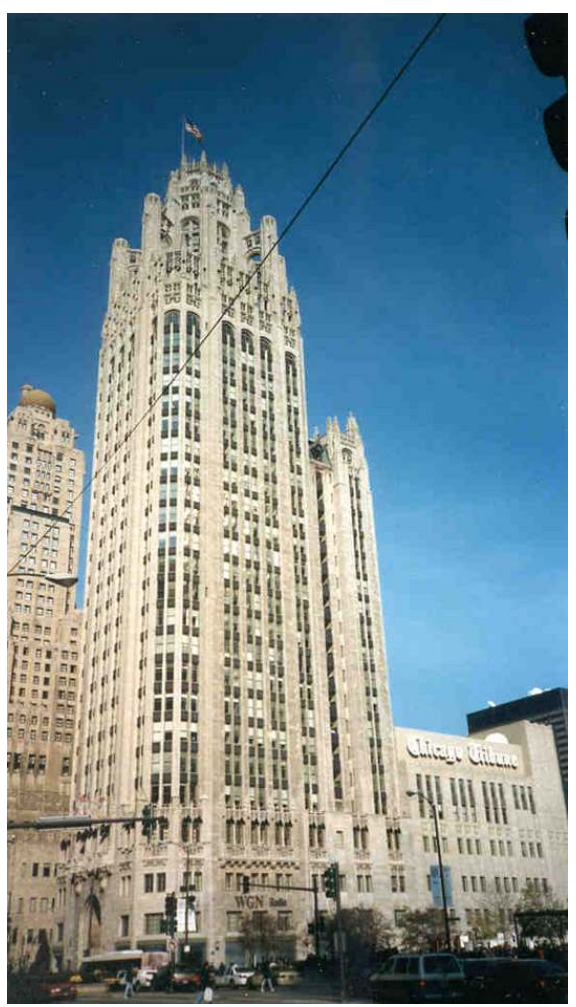

Tribune Tower de Chicago

Phot. Michael P. Gensheimer (c) Michael P. Gensheimer, 2001

La Tribune Tower de Chicago est édifiée entre 1922 et 1925. Ses auteurs, Raymond M. Hood et John Mead Howells, architectes de New York vainqueurs de la compétition (d'où son autre nom de Competition Tower) lancée par le Chicago Tribune, ont explicitement fait référence à la «cathédrale française de Rouen " et construit l'un des plus beaux gratte-ciels néo-gothiques. On peut penser qu'ici le souvenir de Rouen amalgame la Tour de Beurre (avec la base de huit pinacles) avec une plus forte « dose » de Tour couronnée, sensible dans la forme générale plus massée, la couronne plus aérienne et transparente et l'amortissement moins aigu des culées qui évoque les petits dômes de Saint-Ouen.

Information complémentaire : Site City of Chicago

\section{NOTES}

1. Je remercie mes collègues du Service régional de l'inventaire de Haute-Normandie, du Centre et de la Sous-Direction, bibliothèque du Patrimoine et laboratoire photographique, qui ont facilité la préparation de cet article en collectant et numérisant l'illustration. Je remercie tout particulièrement Monsieur Dominique Moufle, architecte en chef des Monuments historiques responsable de Saint-Ouen qui m'a communiqué et autorisé à reproduire les magnifiques relevés 
qu'il vient d'établir sur la tour couronnée Je remercie enfin Renaud Benoit-Cattin qui a relu ce texte et m'a donné quelques encouragements et précisions fort utiles.

2. Pour les amateurs de proportions, il est intéressant de noter que depuis l'achèvement, il y a un peu plus d'un siècle, de la flèche de fonte de fer de la cathédrale projetée par Alavoine en 1828, celle-ci culmine à $154 \mathrm{~m}$ soit presque exactement le double de la hauteur de nos deux tours mesurant, la Tour de Beurre : $77 \mathrm{~m}$ et la Tour couronnée : $79 \mathrm{~m}$. Mais ce rapport symbolique n'existait pas avec l'ancienne flèche de la cathédrale de Robert Becquet, de 1540, qui devait culminer à environ $135 \mathrm{~m}$, ce qui était déjà un record pour cette époque.

3. Il y aurait beaucoup à dire sur le beurre en Normandie à la fin $d u X V^{e}$ siècle, ce qui détournerait quelque peu de cette étude. Il faut peut-être rappeler que ce nec plus ultra des produits laitiers symbolise depuis lors la richesse de la gastronomie normande liée à de nouveaux modes de productions agricoles introduits justement à la fin $\mathrm{du} \mathrm{XV}^{\mathrm{e}}$ siècle et basés sur l'établissement, à côté des céréales, d'une nouvelle économie rurale associant sur les herbages plantés de pommiers à cidre, l'élevage des vaches pour la production de laitages, beurre, crèmes et fromages et de viande de bœuf et de veau qui se consomme beaucoup plus régulièrement dans une société aimant de plus en plus la bonne chère, même pendant le carême ! D'autres pensent de façon peut-être plus sage que ce nom de Tour de Beurre peut lui venir de la couleur de la pierre jaune de l'Oise dont elle est construite, encore inhabituelle à Rouen et qui devait faire penser aux habitants à une énorme motte de beurre, ce qui rejoint l'obsession évoquée plus haut...

4. Toutes les flèches de pierres, et elles furent nombreuses, élevées à Rouen au $\mathrm{XV}^{\mathrm{e}}$ ou au $\mathrm{XVI}^{\mathrm{e}}$ siècle ont aujourd'hui disparu, même si elles avaient fait à l'époque de leur construction l'objet d'une admiration internationale, comme la flèche de Saint-Laurent, l'une des dernières arasées, ou celle de Saint-André dont le pape s'était fait envoyer le relevé.

\section{Notices de la base Mérimée}

5. On sait que la nef fut construite pendant la première moitié du XVI ${ }^{\mathrm{e}}$ siècle et que seul le premier niveau des tours de façades fut implanté. Dans une gravure de Harel, Portail de l'église de Saint-Ouen de Rouen comme il doit être achevé (Paris, B. N. Est. Ve 22 a (II), pl. 19) illustrant l'ouvrage de Dom Pommeraye publié en 1662 sur l'histoire de l'abbaye, on voit tout ce que le projet, du moins tel qu'il était encore conçu au milieu du XVII ${ }^{\mathrm{e}}$ siècle, doit à la Tour couronnée.

6. Rapport de Simon Lenoir et de Jean Willemer, maîtres des oeuvres de maçonnerie et de charpenterie du roi sur les dangers d'écroulement des piliers du transept de l'église Saint-Ouen, 21 janvier 1441, A. D. Seine-Maritime 14 H 440, publié par Jules Quicherat, Documents inédits sur la construction de Saint-Ouen de Rouen, Paris, Bibl. de l'Ecole des Chartes, 1852, pp. 464-476.

7. Cette clef annulaire laisse penser que l'architecte de cette lanterne avait prévu de pouvoir monter des cloches dans un étage supérieur où il avait imaginé un beffroi comme cela se trouve dans d'autres édifices des environs (Jumièges, Saint-Martin-de-Boscherville...), soit à une hauteur exceptionnelle et qui nous paraît aujourd'hui peu réaliste.

8. Ces données sont reprises de Bauchal, Ch., Nouveau dictionnaire..., Paris, 1887, p. 856.

9. Dominique Hervier, notice sur Saint-Gatien de Tours dans le Guide du patrimoine de la région Centre..., p. 487-496.

10. Rappelons qu'il était alors curé de Saint-Maclou de Rouen d'où il pouvait à loisir suivre les deux chantiers qui ont pu inspirer Verneuil. Il n'eut pas le même projet pour Saint Maclou, alors couronné par une flèche de charpente, la flèche de pierre actuelle étant une oeuvre magnifique de Barthélémy achevée vers 1875.

11. Voir : Coupe de la tour de Notre-Dame à Rouen par Pierre-Adrien Pâris. Dessin au crayon, H 1,$06 ; \mathrm{L}$ 0,42, Besançon, bibliothèque municipale. 


\section{RÉSUMÉS}

La Tour de Beurre de la cathédrale et la Tour couronnée de l'abbaye Saint-Ouen se côtoient dans le ciel de Rouen depuis la fin du Moyen Age, et présentent de nombreuses similitudes. Sans prétendre apporter une connaissance nouvelle et complète sur la genèse, l'analyse et la postérité de ces chefs-d'oeuvre, cette étude modeste fait le point des connaissances et situe ces fameux morceaux d'architecture dans le riche contexte des édifices flamboyants du début de la Renaissance à Rouen.

Les deux célèbres tours sont présentées dans leurs rapports avec les grandes églises qu'elles complètent et situées parmi quelques monuments plus ou moins éloignés dans l'espace et dans le temps, qui ont manifestement subi leur très forte influence.

The Butter-tower of the cathedral and the Crowned-tower of the abbey of Saint-Ouen which rise together, since the middle-ages, into the sky at Rouen, have many features in comon. This paper will not add new elements nor pretend to complete genesis, analysis and development of these master-pieces, but, with reference to existing knowledge, places these two important buildings in the context of "flamboyante architecture" at the beginning of the Renaissance period in Rouen.

\section{INDEX}

Mots-clés : inventaire général, en ligne, journal, revue électronique, revue numérique, périodique, patrimoine, histoire de l'art, France, Haute-Normandie, Rouen, architecture religieuse, $15 \mathrm{e}$ siècle, $16 \mathrm{e}$ siècle

Keywords : on line, electronic journal, ejournal, heritage, history of art, France, Normandy, Rouen, religious structures, religious communities, XVth century, XVIth century

\section{AUTEUR}

\section{FRANÇOIS VERDIER}

Conservateur en chef du patrimoine, Inspecteur de l'Inventaire, Direction de l'Architecture et du Patrimoine 3, place de Valois 75001 Paris. francois.verdier@culture.fr 